\title{
A functional genomics strategy reveals clockwork orange as a transcriptional regulator in the Drosophila circadian clock
}

\author{
Akira Matsumoto, ${ }^{3,9,11}$ Maki Ukai-Tadenuma,, ${ }^{1,9}$ Rikuhiro G. Yamada, ${ }^{1,9}$ Jerry Houl, ${ }^{7,8}$ \\ Kenichiro D. Uno, ${ }^{2}$ Takeya Kasukawa, ${ }^{2}$ Brigitte Dauwalder, ${ }^{8}$ Taichi Q. Itoh, ${ }^{4}$ Kuniaki Takahashi, ${ }^{6}$ \\ Ryu Ueda, ${ }^{6}$ Paul E. Hardin, ${ }^{7}$ Teiichi Tanimura, ${ }^{4}$ and Hiroki R. Ueda ${ }^{1,2,5,10}$ \\ ${ }^{1}$ Laboratory for Systems Biology, Center for Developmental Biology, RIKEN, Chuo-ku, Kobe, Hyogo 650-0047, Japan; \\ ${ }^{2}$ Functional Genomics Unit, Center for Developmental Biology, RIKEN, Chuo-ku, Kobe, Hyogo 650-0047, Japan; ${ }^{3}$ Center for \\ Research and Advancement in Higher Education, Kyushu University, Ropponmatu, Fukuoka 810-8560, Japan; ${ }^{4}$ Department \\ of Biology, Faculty of Science, Kyushu University, Ropponmatu, Fukuoka 810-8560, Japan; ${ }^{5}$ Department of Bioscience, \\ Graduate School of Science, Osaka University, Toyonaka, Osaka 560-0043, Japan; ${ }^{6}$ Genetic Strains Research Center, \\ National Institute of Genetics, Mishima, Shizuoka 411-8540, Japan; ${ }^{7}$ Department of Biology and Center for Research on \\ Biological Clocks, Texas A\&M University, College Station, Texas 77843, USA; ${ }^{8}$ Department of Biology and Biochemistry, \\ University of Houston, Houston, Texas 77204, USA
}

The Drosophila circadian clock consists of integrated autoregulatory feedback loops, making the clock difficult to elucidate without comprehensively identifying the network components in vivo. Previous studies have adopted genome-wide screening for clock-controlled genes using high-density oligonucleotide arrays that identified hundreds of clock-controlled genes. In an attempt to identify the core clock genes among these candidates, we applied genome-wide functional screening using an RNA interference (RNAi) system in vivo. Here we report the identification of novel clock gene candidates including clockwork orange (cwo), a transcriptional repressor belonging to the basic helix-loop-helix ORANGE family. cwo is rhythmically expressed and directly regulated by CLK-CYC through canonical E-box sequences. A genome-wide search for its target genes using the Drosophila genome tiling array revealed that cwo forms its own negative feedback loop and directly suppresses the expression of other clock genes through the E-box sequence. Furthermore, this negative transcriptional feedback loop contributes to sustaining a high-amplitude circadian oscillation in vivo. Based on these results, we propose that the competition between cyclic CLK-CYC activity and the adjustable threshold imposed by CWO keeps E-box-mediated transcription within the controllable range of its activity, thereby rendering a Drosophila circadian clock capable of generating high-amplitude oscillation.

[Keywords: Functional genomics; circadian clock; Drosophila; in vivo RNAi; genome tiling array; CHIP-on-chip]

Supplemental material is available at http://www.genesdev.org.

Received March 14, 2007; revised version accepted May 15, 2007.

The core molecular mechanism that mediates circadian oscillator function in Drosophila is thought to be composed of two interlocked transcriptional feedback loops: a period (per)/timeless (tim) loop as a negative loop, and a Clock $(C l k) /$ cycle (cyc) loop as a positive one (Hardin 2005). While the framework of the molecular mechanism of circadian oscillation has been extensively elucidated, we still have not identified all the molecules required for feedback loop function. There have been sev-

\footnotetext{
${ }^{9}$ These authors contributed equally to this work.

Corresponding authors.

${ }^{10}$ E-MAIL uedah-tky@umin.ac.jp; FAX 81-78-306-3194.

${ }^{11}$ E-MAIL akirarcb@mbox.nc.kyushu-u.ac.jp; FAX 81-92-726-4641.

Article published online ahead of print. Article and publication date are

online at http://www.genesdev.org/cgi/doi/10.1101/gad.1552207.
}

eral approaches to study how gene expression is globally regulated by the Drosophila clock mechanism using the subtractive cDNA library screening (Van Gelder et al. 1995; Van Gelder and Krasnow 1996; Rouyer et al. 1997), differential display of RT-PCR (Blau and Young 1999), and genome-wide expression trap screening using luciferase reporter insertions (Stempfl et al. 2002). Recently, high-density oligonucleotide probe arrays have been developed and applied as one of the most comprehensive methods to evaluate how gene expression is regulated by the circadian clock on a genome-wide basis /ClaridgeChang et al. 2001; McDonald and Rosbash 2001; Ceriani et al. 2002; Lin et al. 2002; Ueda et al. 2002).

Although hundreds of clock-controlled genes were identified in Drosophila, the functional classification of these genes as components of the circadian clock mecha- 
nism remained for future studies. As a first step, core clock genes, which encode critical components of feedback loops, must be distinguished from clock-regulated output genes. An effective way of distinguishing core clock genes from output genes is to observe the circadian phenotypes of mutants in these genes. Such a mutant screening strategy has significantly facilitated analysis of the clock mechanism in Drosophila (Konopka and Benzer 1971; Sehgal et al. 1994; Allada et al. 1998; Price et al. 1998; Rutila et al. 1998; Blau and Young 1999). However, there are some disadvantages with this strategy when we apply it at a genome-wide level. In particular, mutants are not available for many candidate genes, and even if they are, many of these mutants are lethal during development (Blau and Young 1999). In this study, we established a new functional genomic experimental strategy that can overcome the disadvantages of more traditional mutant screening. This strategy allowed us to identify additional core clock gene candidates. Further analyses revealed that CG17100, one of these candidates, is able to regulate its own expression to form a third feedback loop in the Drosophila clock that interlocks with the two existing loops. Our strategy of combining RNA interference (RNAi) screening and chromatin immunoprecipitation (ChIP)-on-chip assays using genome tiling arrays represents a new functional genomics strategy for analyzing the function and targets of transcription factors.

\section{Results}

Genome-wide tissue-specific knockdown analysis in vivo reveals cwo as a clock gene candidate

We previously performed a comprehensive analysis of circadian gene expression in Drosophila heads by profiling whole-genome microarrays (Ueda et al. 2002). This involved selecting a total of 200 genes with rhythmic expression both in light-dark (LD) and constant darkness (DD) conditions (Supplementary Table 1). In order to screen for core clock genes among these candidates, we adopted an RNAi approach by using the double-stranded RNA (dsRNA) of the target gene selectively induced by the Gal4-UAS binary system (Pili-Floury et al. 2004). We established a UAS-inversed repeat (UAS-IR) transgenic strain carrying 500-base-pair (bp) fragments of a target gene in a head-to-head manner into a pUAST-R57 plasmid (Fig. 1A) to express dsRNA for the target gene. The target site of dsRNAi (500-bp fragment) was selected to minimize the possibility of off-target effects as calculated by dsCheck (http://dscheck.rnai.jp) (Naito et al. 2005). We generally established four to five transgenic lines, each of which carries UAS-IR at a different site of the genome, then selected two lines that have no apparent insertional mutation. We mated UAS-IR transgenic flies to the tim(UAS)-gal4 strain (Blau and Young 1999) as a driver to induce a knockdown of the target gene's expression by RNAi in tim-positive cells, including pacemaker neurons. Any abnormalities in locomotor rhythms of these knockdown flies would reveal the target gene as an important element in maintaining circadian rhythmicity.
We first checked whether our knockdown strategy could be applied to screen for clock genes with circadian expression, namely, per, tim, vri, and Clk (Hardin 2005). We established two independent transgenic lines of UAS-per-IR and recorded locomotor activity rhythm in tim(UAS)-gal4/UAS-per-IR trans-heterozygotes (Fig. 1B, left). All flies $(n=28)$ from both lines showed arrhythmicity under DD, suggesting that our knockdown strategy worked well in the circadian phenotype. As the per gene knockdown was reported to induce a long period phenotype (Martinek and Young 2000), the effect was more severe in our case than in previous ones. We further checked the effect of RNAi-mediated knockdown on other clock genes (Fig. 1B, left). While 15 out of 25 tim knockdown flies became arrhythmic, the remaining 10 still showed normal rhythmicity. On the other hand, RNAi-mediated knockdown of Clk and vri had little or no effect on locomotor activity rhythms since all flies tested were rhythmic with $\sim 24-h$ periods (data not shown). RNAi works with different efficiencies with different genes. Perhaps our vri-IR or Clk-IR strains showed little decrease in CLK and VRI levels, respectively. Alternatively, compared with perturbations of per and tim, the fly clock system might be relatively resistant to perturbation of Clk and vri. In fact, moderate decreases in CLK may have little impact on feedback loop function since CLK activity is controlled at the post-transcriptional level (Yu et al. 2006), and heterozygous vri mutants show only modest increases of Clk mRNA /Glossop et al. 2003).

We screened an additional 133 genes and isolated five as novel core clock gene candidates (Fig. 1B, right; Table 1). The deduced functions of the gene products of these candidates are very diverse; a transcription factor (CG17100), an ABC transporter (E23), a chromatin remodeling factor (Iswi), and a factor affecting cell proliferation (prod). The function of the one remaining gene product (CG5273) remains unknown. Among these candidates, we focused on the CG17100 gene, which has been renamed clockwork orange (cwo) (Lim et al., 2007), since the locomotor phenotype was the strongest and most stable among all of the candidates, with one cwo RNAi transgenic line (cwo-IR2) showing a long period phenotype $(26.01 \pm 0.07 \mathrm{~h}, n=37)$ (Fig. 1B). In addition, three out of 40 flies in this line were arrhythmic (Table 1). Although CG17100 was previously annotated as sticky chordotonals 1 (stich1), Dubruille et al. (2002) showed that the stich1 mutation was mapped to jumu, a neighboring gene to CG17100. Thus, we used cwo as a new gene name for CG17100. To confirm that the CWO protein level is reduced in the head of the knockdown flies, an antibody was raised against full-length CWO protein (see Materials and Methods). Western blot analyses with antisera against CWO protein confirmed that the expression of CWO protein reduced to less than onequarter level of wild type (Supplementary Fig. 2A,C). The other line (cwo-IR3), which carries a UAS-IR insertion at a different position of the genome from that of cWO-IR2, also showed a long period phenotype $(25.61 \pm 0.20 \mathrm{~h}$, $n=8$ ). We also observed a similar circadian phenotype in 
A

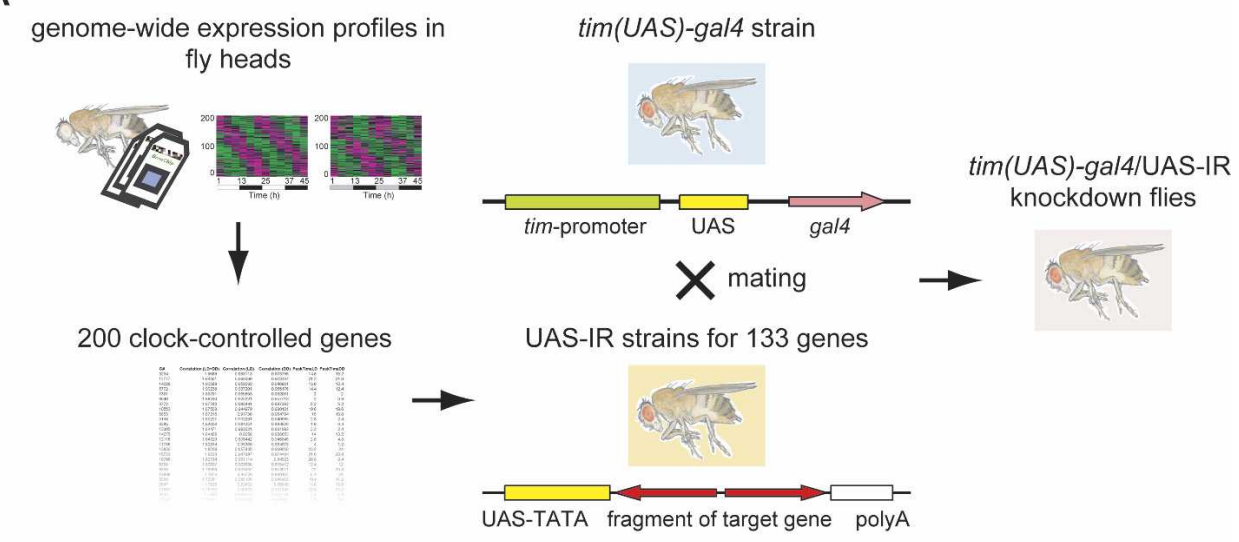

B
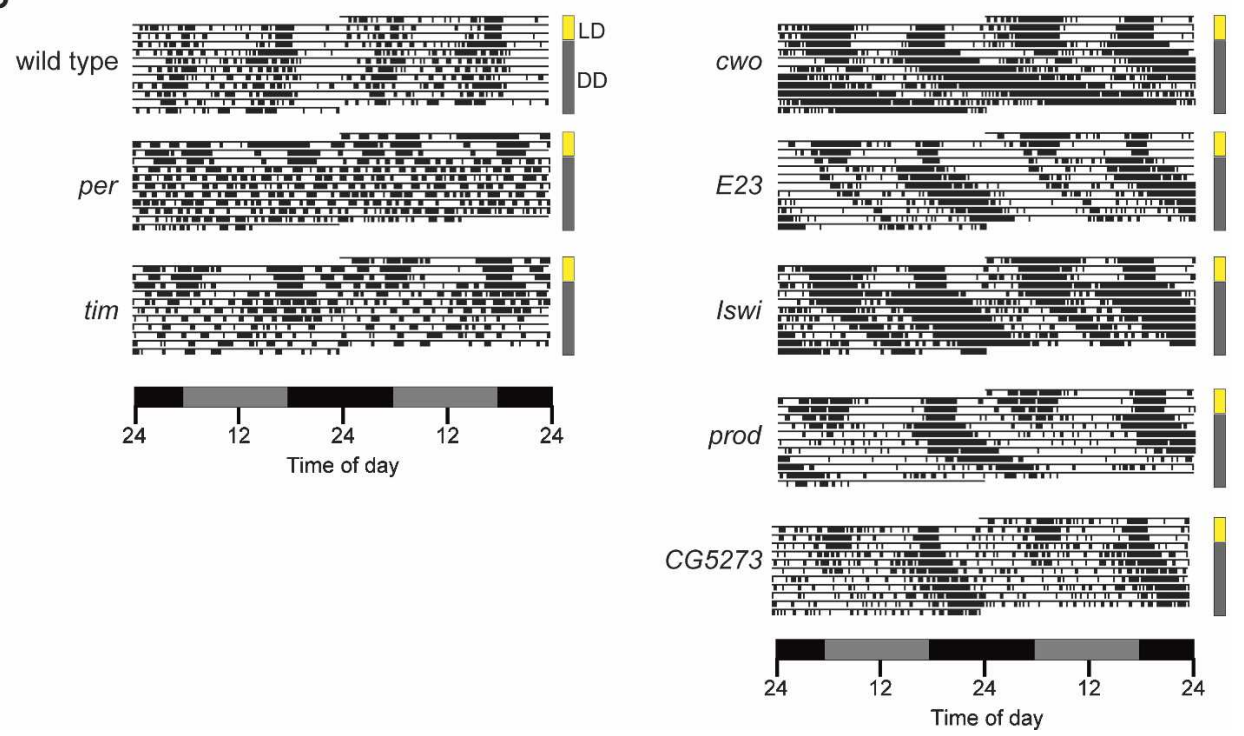

Figure 1. A functional genomics strategy revealed cwo as a clock component. (A) Genome-wide tissue-specific knockdown analysis of clock-controlled genes in Drosophila. UAS-IR transgenic lines to express dsRNA for the target gene under the control of UAS were established (Pili-Floury et al. 2004). Usually two independent insertion lines are established for one target gene. Each of the UAS-IR lines was mated to tim(UAS)-gal4 to drive the expression of dsRNA specifically within clock cells. The locomotor activity of RNAi transgenic flies for 133 candidates among 200 clock-controlled genes was recorded in DD. UAS-TATA sequence or UAS sequence (yellow rectangle), SV40 late polyA site (white rectangle), 500-bp fragment of a target gene (red arrow), a tim promoter region (green rectangle), and gal4 gene (pink arrow) are represented. (B) Typical locomotor activity in wild-type, knockdown flies of known clock genes (left) and five new candidates (right). The names of the knocked-down genes are described at each actogram.

cwo RNAi transgenic flies with pdf-gal4 (Renn et al. 1999) and gal $^{1118}$ strains (Malpel et al. 2002) as drivers. As GAL4 is commonly expressed in the pacemaker neurons of a fly brain in three different driver strains (Blau and Young 1999; Renn et al. 1999; Malpel et al. 2002), the target cells of the circadian phenotype in cwo RNAi transgenic flies are thought to be the central pacemaker neurons.

\section{cwo is a new clock gene}

To exclude the possibility of off-target effects of RNAi, we also monitored the phenotype of a cwo mutant strain (f05073; Exelixis collection, Harvard University) that has a transposon insertion in the first $c$ wo intron. CWO ex- pression was drastically reduced in homozygous $f 05073$ flies (Fig. 2C; Supplementary Fig. 2B,D). Homozygous f05073 flies had long period rhythms similar to cwo RNAi transgenic flies, and a substantial portion of them were arrhythmic (data not shown; see also accompanying paper by Kadener et al. 2007). In addition, two independent strains with overexpression of a dominant-negative type of CWO protein $(\Delta b-1$ and $\Delta b-4$; see also Materials and Methods for details) also showed abnormal rhythmicity similar to 05073 ; that is, a part of them became arrhythmic $(n=15$ in $\Delta b-1$ and $n=3$ in $\Delta b-4)$ while the remaining showed a longer period $(25.16 \pm 0.12$ h, $n=9$ in $\Delta b-1 ; 24.86 \pm 0.09 \mathrm{~h}, n=18$ in $\Delta b$-4) (Table 1 ; Supplementary Fig. 1). In both strains, arrhythmicity oc- 
Table 1. Free-running periods of wild-type, knockdown, and cwo mutant flies

\begin{tabular}{|c|c|c|c|c|}
\hline Gal4 driver & IR line & $\begin{array}{c}\text { Period } \\
(\text { mean } \pm \text { SEM }) \\
(\mathrm{h})\end{array}$ & $\mathrm{N}_{\mathrm{R}}$ & $\mathrm{N}_{\mathrm{A}}$ \\
\hline Wild-type & & $23.83 \pm 0.07$ & 26 & 0 \\
\hline \multirow[t]{12}{*}{$\operatorname{tim}(U A S)$-gal4 } & cwo-IR2 & $26.01 \pm 0.07^{\mathrm{a}}$ & 37 & 3 \\
\hline & $c W O-I R 3$ & $25.61 \pm 0.20^{\mathrm{a}}$ & 8 & 0 \\
\hline & E23-IR1 & $24.73 \pm 0.12^{\mathrm{a}}$ & 25 & 0 \\
\hline & E23-IR2 & $24.81 \pm 0.19^{\mathrm{a}}$ & 11 & 0 \\
\hline & CG5273-IR1 & $24.30 \pm 0.11^{\mathrm{a}}$ & 13 & 0 \\
\hline & CG5273-IR2 & $24.52 \pm 0.09^{\mathrm{a}}$ & 13 & 0 \\
\hline & CG5273-IR3 & $24.93 \pm 0.20^{\mathrm{a}}$ & 6 & 0 \\
\hline & Iswi-IR1 & $24.48 \pm 0.02^{\mathrm{a}}$ & 30 & 2 \\
\hline & Iswi-IR2 & $24.86 \pm 0.07^{\mathrm{a}}$ & 15 & 0 \\
\hline & prod-IR1 & $24.19 \pm 0.19^{\mathrm{a}}$ & 8 & 1 \\
\hline & prod-IR2 & $25.48 \pm 0.09^{a}$ & 6 & 0 \\
\hline & prod-IR3 & $24.25 \pm 0.11^{\mathrm{a}}$ & 11 & 1 \\
\hline \multirow[t]{5}{*}{ pdf-gal4 } & cwo-IR2 & $25.91 \pm 0.30^{\mathrm{a}}$ & 14 & 26 \\
\hline & cWO-IR3 & $25.13 \pm 0.08^{\mathrm{a}}$ & 27 & 4 \\
\hline & E23-IR1 & $25.01 \pm 0.09^{\mathrm{a}}$ & 15 & 0 \\
\hline & $\Delta b-1$ & $25.16 \pm 0.12^{\mathrm{a}}$ & 9 & 15 \\
\hline & $\Delta b-4$ & $24.86 \pm 0.09^{\mathrm{a}}$ & 18 & 3 \\
\hline$g a 1^{1118}$ & $c W O-I R 3$ & $25.99 \pm 0.22^{\mathrm{a}}$ & 13 & 0 \\
\hline
\end{tabular}

$\left(\mathrm{N}_{\mathrm{R}}\right)$ Number of rhythmic flies; $\left(\mathrm{N}_{\mathrm{A}}\right)$ number of arrhythmic flies.

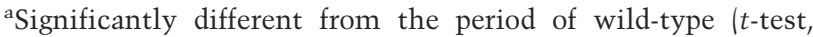
$p<0.05$.

curred after 2-4 d in DD. These behavioral experiments show that the quantitative and qualitative changes in CWO have clear effects on circadian rhythms. Based on these results, we conclude that decreased activity of the CWO protein leads to the abnormalities in the circadian clock.

cwo encodes a basic helix-loop-helix (bHLH)-ORANGE family transcription factor

cwo encodes a candidate transcriptional repressor, with a length of 685 amino acids, belonging to the bHLHORANGE family (Fig. 2A; Davis and Turner 2001). The CWO protein, however, lacks the tetrapeptide domain that generally exists in the $\mathrm{C}$ terminus of Hey, hairy, or E(spl) subfamilies in the bHLH-ORANGE family (Davis and Turner 2001) and belongs to the Stra13 subfamily. We found that the CWO protein is not only a structural ortholog of DEC1 and DEC2 proteins in mammals but also has a highly conserved sequence with the HES5 protein. DEC1 and DEC2 also lack the tetrapeptide domain (Davis and Turner 2001), are rhythmically expressed, and are capable of negatively regulating transcription of clock-controlled genes through the E-boxes in mammals (Honma et al. 2002; Grechez-Cassiau et al. 2004). We were also interested to find that Hes5 has a rhythmic expression in the suprachiasmatic nucleus (SCN) (Supplementary Fig. 3). Hence, the three structural homologs of CWO protein in mammals can be regarded as being associated with the circadian clock, although circadian phenotypes of mutants in these genes have not yet been reported in vivo.

cwo mRNA is rhythmically expressed in the fly head, and CWO protein is expressed in the oscillator neurons

Our quantitative RT-PCR (Q-PCR) measurement revealed that temporal expression of cwo rhythmically changed in LD and DD in wild-type flies, peaking very closely in phase with per and tim, which is consistent with previous genome-wide expression analysis (Fig. 2B; McDonald and Rosbash 2001; Ceriani et al. 2002; Lin et al. 2002; Ueda et al. 2002). The phase of cwo mRNA cycling and lack of rhythmic expression in $\mathrm{per}^{01}$ and $C l k^{I r k}$ mutants (Supplementary Fig. 4) suggest that $c w O$ is regulated by the same molecular mechanism as per and tim. Like other CLK-CYC-dependent genes, per and tim are expressed in six groups of canonical brain oscillator neurons: dorsal lateral neurons $\left(\mathrm{LN}_{\mathrm{d}} \mathrm{s}\right)$, small and large ventral lateral neurons $\left(\mathrm{sLN}_{\mathrm{v}} \mathrm{s}\right.$ and $\mathrm{lLN}_{\mathrm{v}} \mathrm{s}$, respectively), and dorsal neurons 1, 2, and 3 (DN1, DN2, and DN3) (Zerr et al. 1990; Hunter-Ensor et al. 1996). Brains from wild-type flies collected at Zeitgeber time (ZT) 21 were coimmunostained with antisera against $\mathrm{CWO}$ and the oscillator neuron marker PER. CWO immunoreactivity was detected in all three groups of DNs (Fig. 2C, panels 1-3). Since PER is found in nuclei at this time (Curtin et al. 1995), the overlap in CWO and PER immunostaining indicates that CWO is primarily or completely nuclear (Fig. 2C, panels 3,3m,6,6m). Likewise, CWO immunoreactivity is detected in nuclei from $\operatorname{sLN}_{\mathrm{v}} \mathrm{s}, \mathrm{lLN}_{\mathrm{v}} \mathrm{s}$, and $\mathrm{LN}_{\mathrm{d}} \mathrm{s}$ (Fig. 2C, panels 4-6). This pattern of CWO immunostaining is consistent with reporter gene expression from an apparent $c$ wo enhancer trap line, R32 (Shafer et al. 2006). In homozygous f05073 mutants, CWO is not detected in any of the six canonical groups of brain oscillator neurons (Fig. 2C, panels $7-12 \mathrm{~m}$ ), consistent with Western results (Supplementary Fig. 2). Given that $\mathrm{sLN}_{\mathrm{v}} \mathrm{s}$ are necessary and sufficient for behavioral rhythms (Renn et al. 1999; Grima et al. 2004), our results suggest that cwo functions in brain oscillator neurons to maintain behavioral rhythms.

\section{cwo is controlled by CLK through canonical E-boxes}

Oscillatory expression of cwo mRNA became arrhythmic in per $^{01}$ and $C l k^{I r k}$ mutants (Supplementary Fig. 4), and its expression profile was similar to those of per and tim mRNA in per ${ }^{01}$ and $C l k^{I r k}$ mutants (Fig. 3A). These results strongly suggested that cwo is induced by CLKCYC as in per and tim (Darlington et al. 1998). As CLKCYC is known to bind E-boxes in the per and tim promoter regions (Hao et al. 1999; Darlington et al. 2000; McDonald et al. 2001), we searched for E-boxes in the cwo promoter region (Fig. 3B). The 2.8-kb promoter region of cwo contains five canonical E-boxes (CACGTG), five $E^{\prime}$-boxes (CACGTT), and 16 noncanonical E-box sequences (CANNTG other than CACGTG). The observed frequencies of these elements within the 2.8 - $\mathrm{kb}$ pro- 
A

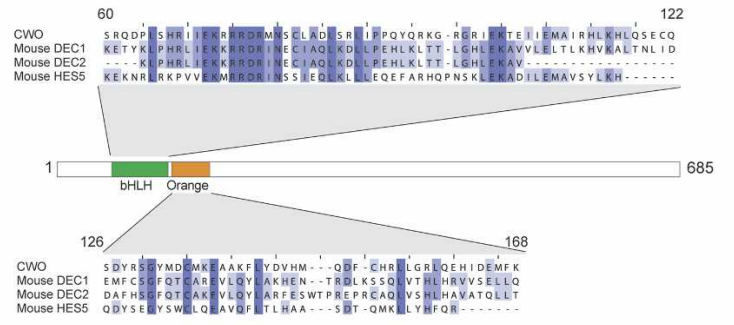

B
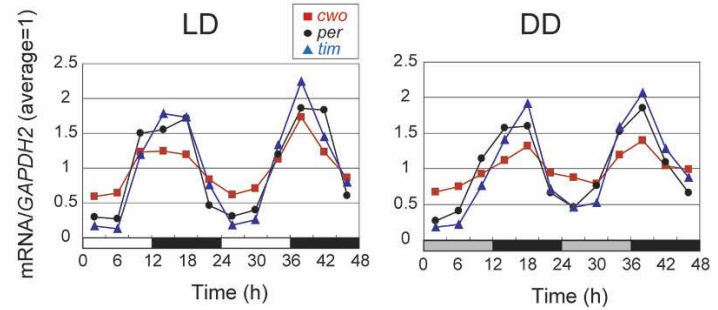

C
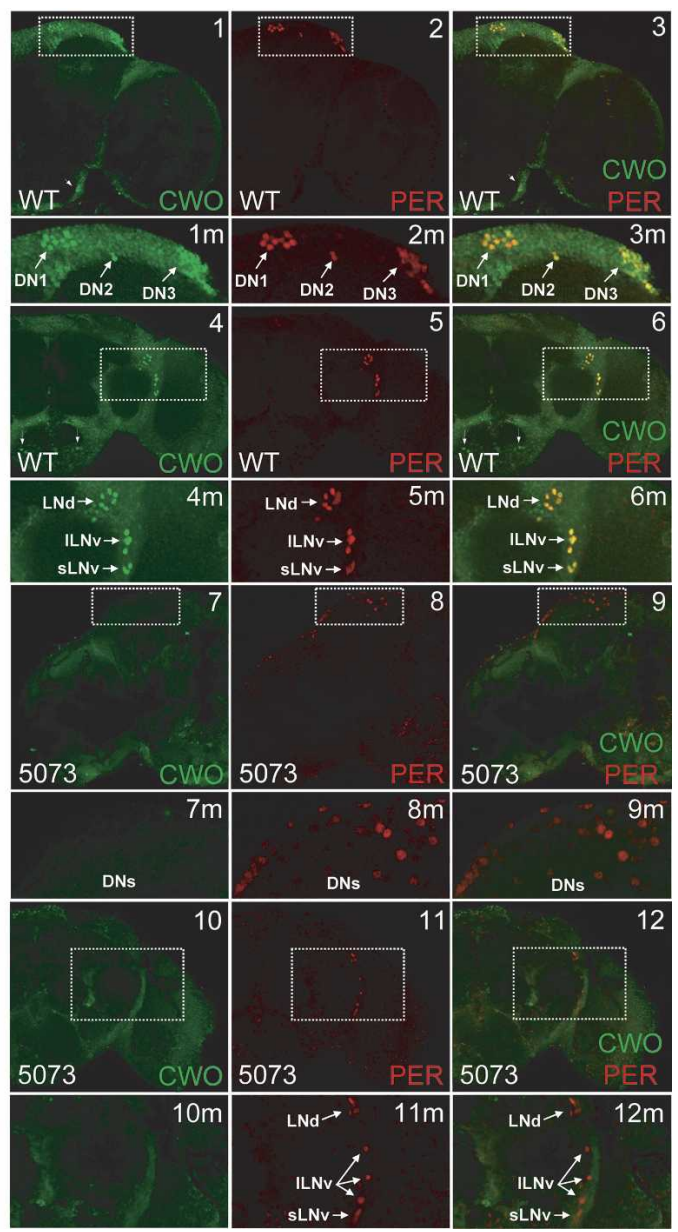

Figure 2. Temporal and spatial expression pattern of CWO protein. (A) cwo encodes a bHLH-ORANGE family protein. The CWO protein, 685 amino acids in length, is multiply aligned with mouse DEC1, DEC2, and HES5 proteins. Conserved amino acids in bHLH (green) and ORANGE (orange) domains are marked by blue and gaps are represented as "-". (B) Temporal expression profiles of $c w O$ (red square), per (black circle), and tim (blue triangle) mRNA in wild-type flies under LD and DD. Relative mRNA levels of the indicated genes were measured using a Q-PCR assay. GAPDH2 was used as an internal control. Data were normalized so that the average copy number $(n=2)$ over 12 time points is 1.0. (C) Spatial expression pattern of CWO in adult brains. (Panel 1) CWO

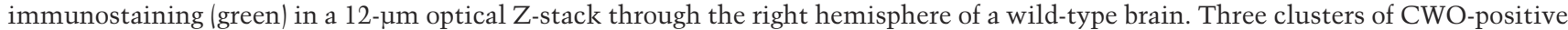
cells are detected in the dorsal brain. A magnified view of the boxed region is shown in panel 1m. Arrows denote DN1s, DN2s, and DN3s. Arrowheads denote additional CWO immunostaining. (Panel 2) PER immunostaining (red) in the same region shown in panel 1. Three clusters of PER-positive cells are detected in the dorsal brain. A magnified view of the boxed region is shown in panel $2 \mathrm{~m}$. Arrows denote the same cells described in panel $1 \mathrm{~m}$. (Panel 3) CWO and PER coimmunostaining in the same region shown in panels 1 and 2. Colocalization of CWO and PER immunofluorescence in this superimposed dual laser image is shown in yellow. A magnified view of the boxed region is shown in panel $3 \mathrm{~m}$. Arrows denote the same cells described in panels $1 \mathrm{~m}$ and $2 \mathrm{~m}$. Arrowheads denote CWO staining in cells not expressing PER. (Panel 4) CWO immunostaining in a 22-um optical Z-stack through the right hemisphere of a wild-type brain. Three clusters of CWO-positive cells are detected in the lateral brain. A magnified view of the boxed region is shown in panel $4 \mathrm{~m}$. Arrows denote clusters of $\mathrm{LN}_{\mathrm{d}} \mathrm{s}, \mathrm{LN}_{\mathrm{v}} \mathrm{s}$, and $\mathrm{sLN}_{\mathrm{v}} \mathrm{s}$. Arrowheads denote additional CWO immunostaining. (Panel 5) PER immunostaining in the same region shown in panel 4. Three clusters of PER-positive cells are detected in the lateral brain. A magnified view of the boxed region is shown in panel 5m. Arrows denote the same cells described in panel $4 \mathrm{~m}$. (Panel 6) CWO and PER coimmunostaining in the same region shown in panels 4 and 5. Colocalization of CWO and PER immunofluorescence is shown in yellow. A magnified view of the boxed region is shown in panel $6 \mathrm{~m}$. Arrows denote the same cells described in panels $4 \mathrm{~m}$ and $5 \mathrm{~m}$. Arrowheads denote CWO staining in cells not expressing PER. (Panel 7) CWO immunostaining in an 8- $\mu$ m optical Z-stack through the right hemisphere of a $f 05073$ mutant brain. No specific CWO immunostaining is detected. A magnified view of the boxed dorsal brain region is shown in panel 7m. (Panel 8) PER immunostaining in the same region shown in panel 7. PER-positive DNs are detected in the dorsal brain. A magnified view of the boxed region is shown in panel 8m. (Panel 9) CWO and PER coimmunostaining in the same region shown in panels 7 and 8. Only PER immunofluorescence in DNs is seen in this superimposed dual laser image. A magnified view of the boxed region is shown in panel $9 \mathrm{~m}$. (Panel 10) CWO immunostaining in a 24-pm optical Z-stack through the right hemisphere of a $f 05073$ mutant brain. No specific CWO immunostaining is detected. A magnified view of the boxed region is shown in panel 10m. (Panel 11) PER immunostaining in the same region shown in panel 10. Three clusters of PER-positive cells are detected in the lateral brain. A magnified view of the boxed region is shown in panel $11 \mathrm{~m}$. Arrows denote clusters of $\operatorname{LN}_{\mathrm{d}} \mathrm{s}, \operatorname{lLN}_{\mathrm{v}} \mathrm{s}$, and sLN $\mathrm{v}$. (Panel 12) CWO and PER coimmunostaining in the same region shown in panels 10 and 11. Only PER immunofluorescence is detected. A magnified view of the boxed region is shown in panel $6 \mathrm{~m}$. Arrows denote the same cells described in panel $11 \mathrm{~m}$. 
Matsumoto et al.

A
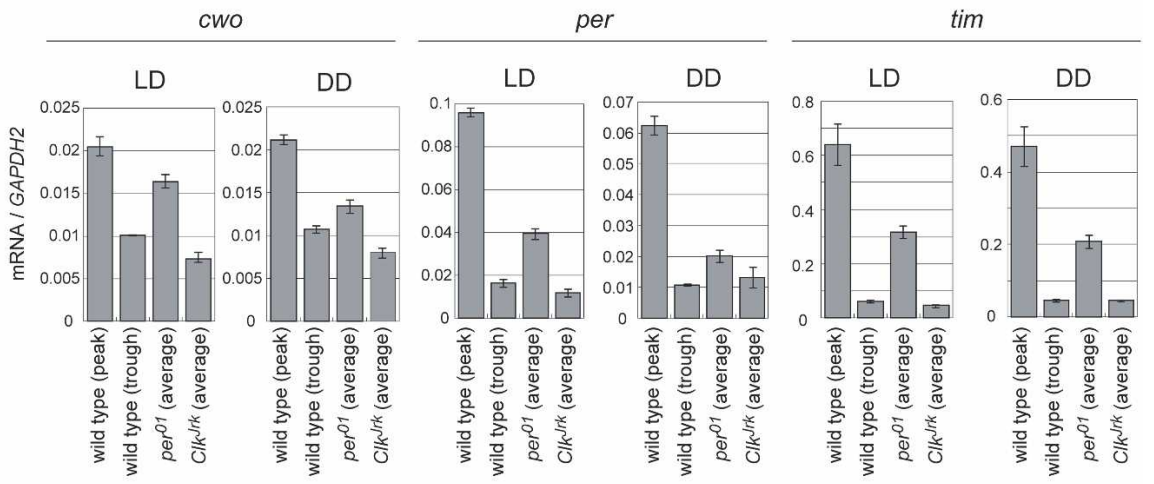

B

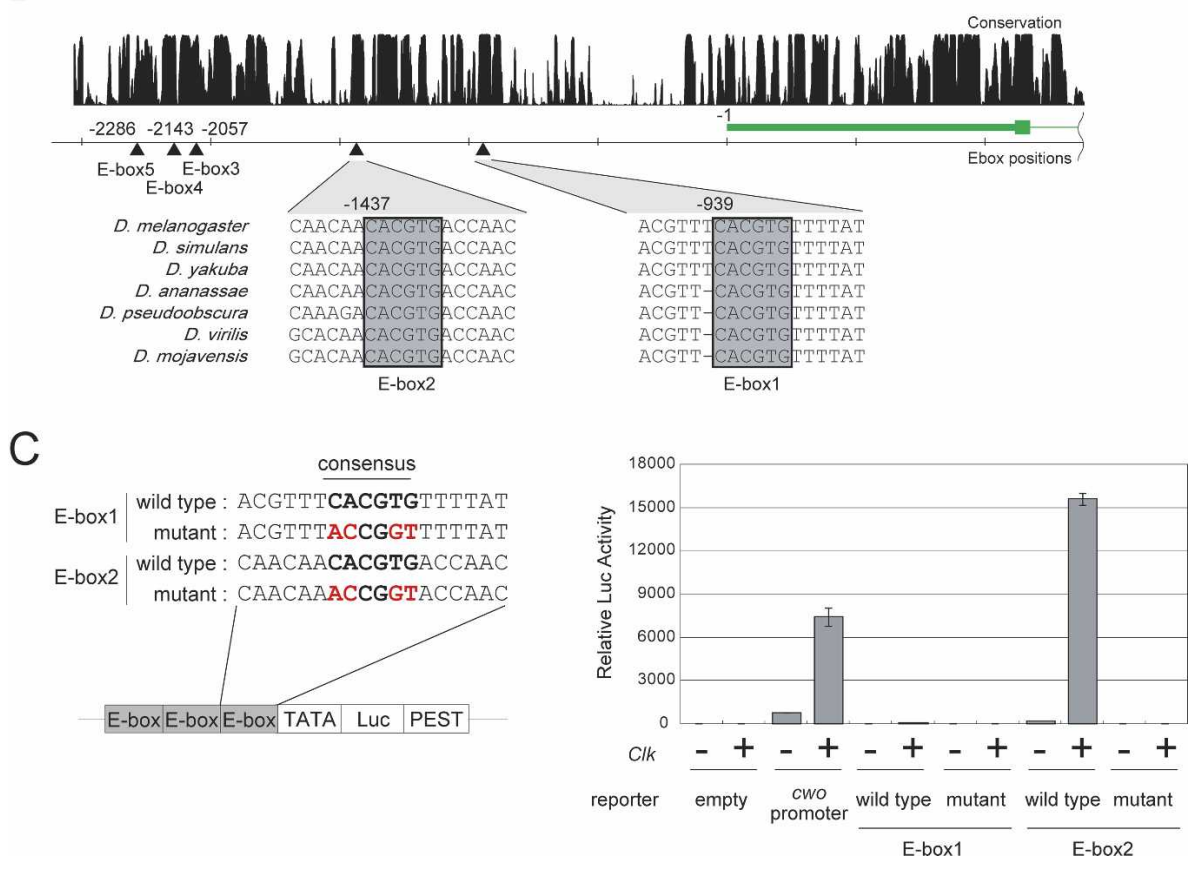

Figure 3. cwo is directly controlled by circadian clock. (A) The averaged expression levels of cwo, per, and tim mRNA in wild-type and arrhythmic mutant flies $\left(\right.$ per $^{01}$ and $\left.C l k^{I r k}\right)$. Temporal expression profiles in mutant flies are shown in Supplementary Figure 4. (B) Evolutionarily conserved E-box sequences in $c w 0$ promoter region. Relative positions of E-boxes to the transcription start site of $c w 0$ are indicated. Multiple alignments of seven Drosophila species and its conservation score are represented (University of California at Santa Cruz Genome Browser Database [Hinrichs et al. 2006] version dm2). (C) The $c$ wo promoter is activated by CLK. (Top panel) The three tandem repeats of an 18-bp fragment containing wild-type and mutant E-boxes were fused to a TATA sequence driving a destabilized luciferase (dLuc) reporter gene. Well-conserved base pairs (bold) and mutated base pairs (red) are also indicated. Relative luciferase activities of $c$ wO promoter, wild-type, and mutant E-box reporters in the presence of 0 and $100 \mathrm{ng}$ of Clk are shown in the bottom panel. Signals were normalized with Rluc activity. Error bars represent the SEM $(n=3)$.

moter region is significantly higher than expected for random DNA sequences $(p<0.0001$ for a canonical E-box, $p=0.0101$ for an $\mathrm{E}^{\prime}$-box, $p=0.0682$ for a noncanonical E-box, and $p=0.0008$ for all of these elements). These elements were also observed within the $7.3-\mathrm{kb}$ first intron of $c$ wo with significantly high frequencies $(p=0.0004$ for six canonical E-boxes, $p=0.0024$ for 10 $\mathrm{E}^{\prime}$-boxes, $p=0.1338$ for 35 noncanonical E-boxes, and $p=0.0020$ for the total of them all). It is noteworthy that only the cwo gene among 13,776 genes $(20,406$ transcripts) in the Drosophila genome has as many as five E-boxes within the 2.8-kb promoter region. Among these
E-box related elements in the cwo gene's promoter, we found two canonical E-boxes (E-box1 and E-box2) to be highly conserved among several Drosophila species (Fig. $3 B)$. We therefore examined whether CLK-CYC can induce gene expression through these E-boxes in Drosophila culture cell S2. Cotransfection with Clk strongly induced luciferase expression with E-box2, while E-box1 and their mutants showed little induction, suggesting that cwO can be directly regulated by CLK through Ebox2 (Fig. 3C). Recently, we found in a mammalian circadian clock system that neighboring sequences of canonical E-boxes (CACGTG) are important to function as 
the clock-controlled element (Y. Kumaki, M. Ukai-Tadenuma, K.D. Uno, J. Nishio, K. Masumoto, M. Nagano, T. Komori, Y. Shigeyoshi, J.B. Hogenesch, and H.R. Ueda, in prep.), which is consistent with previous results in Drosophila showing that sequences flanking the canonical per CRS E-box are required for its function as a clock regulatory element (Lyons et al. 2000). Based on these findings, we can hypothesize that some E-boxes with certain neighboring sequences such as E-box2 in the above are favored by CLK/CYC, and other E-boxes with different neighboring sequences such as E-boxl are favored not by CLK/CYC but by other bHLH transcription factors.

\section{ChIP-on-chip assays reveal that CWO protein binds to canonical E-boxes}

The CWO protein has a bHLH known to act as a DNAbinding domain (Fig. 2A). In an attempt to identify potential targets of CWO protein at the genome-wide level, we performed a ChIP assay using a Drosophila genome tiling array (Manak et al. 2006). In this assay, Flag-tagged CWO protein was induced in S2 cells and immunoprecipitated with its target DNA using anti-Flag antibody. The precipitated DNA was then labeled and hybridized to a tiling array. As a background control, we carried out immunoprecipitation using anti-V5 antibody and performed subsequent tiling array experiments in the same way. Potential candidates for binding sites were identified by TileMap (Ji and Wong 2005) as genomic sites where signals from Flag-ChIP were significantly higher than that from V5-ChIP. We were able to do this by comparing the signal intensity between the two sets of tiling arrays, one set for Flag-ChIP and the other set for V5ChIP $(n=2$; two arrays for both sets). The subsequently detected potential binding sites of CWO protein in the Drosophila chromosome are illustrated in Figure 4A. Detailed information on all the detected sites is available online at our Web site (see Materials and Methods). Among the 1512 sites detected, we confirmed that the CWO protein binds to the known clock genes vri and
A

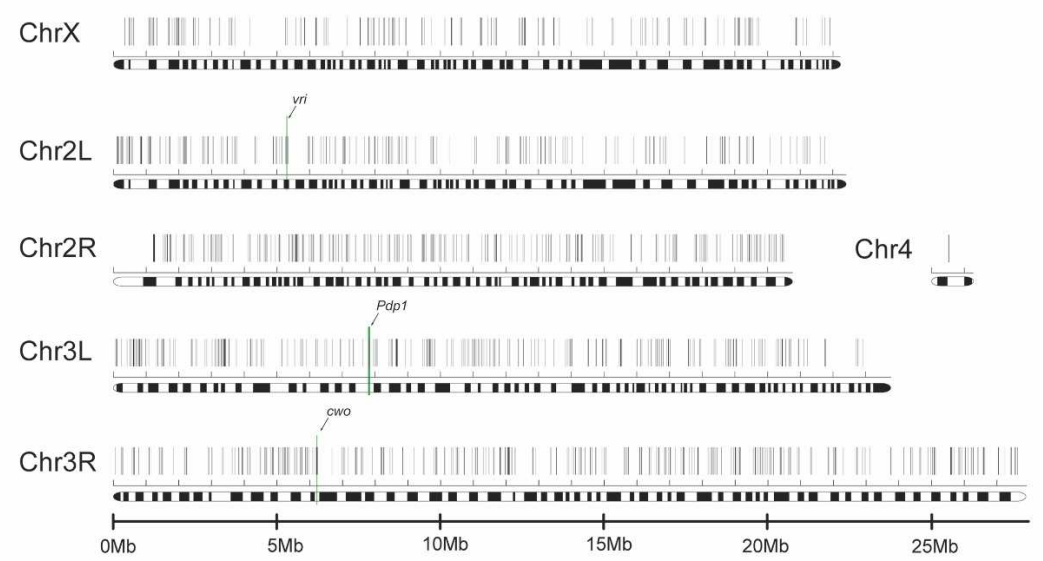

B

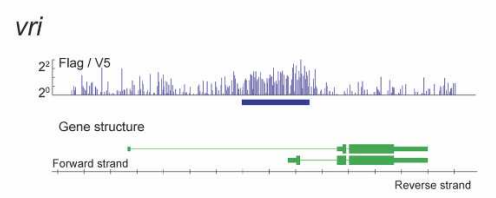

cWo
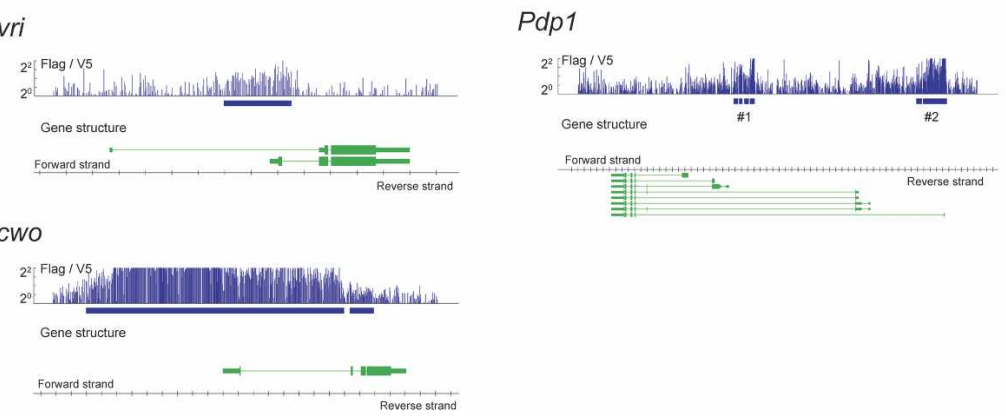

\section{C}
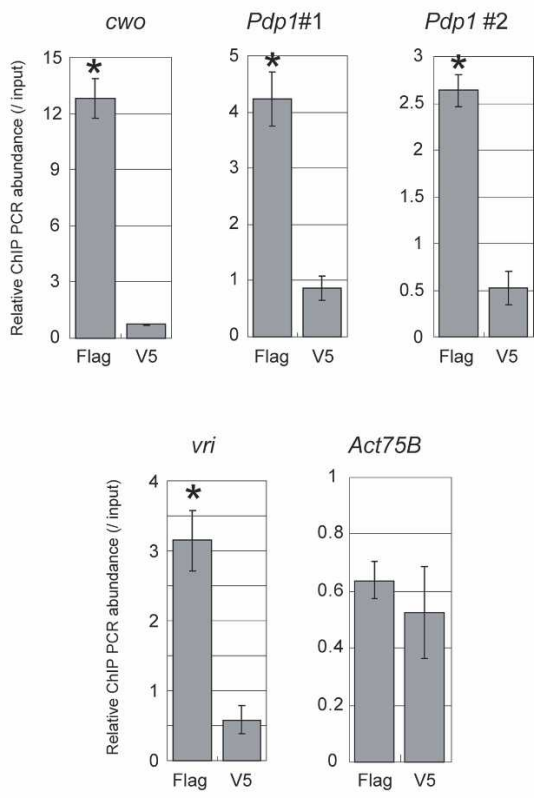

D

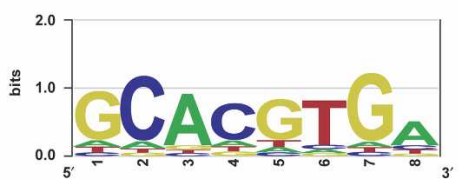

Figure 4. CWO protein directly targets known clock genes. (A) Chromosomal view of potential CWO-binding sites (black vertical bar on each chromosome) identified by ChIP assay on a Drosophila genome tiling array. The locations of vri, Pdp1, and cwo are indicated as a green vertical bar. (B) Close view of potential cwo-binding sites on vri, Pdp1, and cwo genes. (Top panel) The fold changes between signal (Flag) and background (V5) were plotted for each probe with the potential binding sites (blue box). (Bottom panel) Gene structures are also indicated. We identified two independent binding sites in the $P d p 1$ promoter $(P d p 1 \# 1$ and \#2). (C) Independent verification of ChIP experiments. The relative abundance of immunoprecipitated chromosome regions was measured using a Q-PCR assay. Input product (genomic DNA without ChIP) was used as an internal control. Act57B is used as a negative control. Error bars represent the SEM $(n=3)$. cwo, Pdp1, and vri promoters are significantly bound by CWO protein. $\left({ }^{*}\right) p<0.05$ in $t$-test. $(D)$ Canonical E-box (CACGTG) recognized by CWO protein. The DNA sequence overrepresented in potential CWO-binding sites was identified by Weeder and drawn by EnoLOGOS. 
Pdp1, both of which have the E-box sequence in their promoter region (Fig. 4B). We did not detect CWO binding in the promoter regions of per and tim, probably due to the stringent threshold applied to the CWO ChIP analysis, or alternatively reflecting the characteristics of arrhythmic S2 cells that do not normally express per and tim mRNAs. We were also interested to find that CWO protein strongly binds to its own promoter region (Fig. $4 \mathrm{~B})$. The significance of these results was verified by QPCR on each promoter region using the ChIP product as a template (Fig. 4C). Although we cannot exclude the possibility that overexpression in this ChIP experiment might reveal some false-positive interactions, our subsequent bioinformatics search for the consensus DNA sequence recognized by the $\mathrm{CWO}$ protein identified a sequence containing the canonical circadian E-box (CAC GTG) (Fig. 4D), strongly implicating the CWO protein as directly targeting the E-box. As the bHLH-ORANGE family acts as a negative transcription factor, it is possible that the CWO protein not only negatively regulates known clock genes that possess E-boxes in their promoter regions but also its own gene's transcription. This idea led us to the hypothesis that cwo forms an autoregulatory negative feedback loop.

cwo negatively regulates the expression of clock genes and forms an autoregulatory feedback loop

To test whether cwo forms an autoregulatory negative feedback loop, we performed transcription assays using a luciferase reporter gene in S2 cells. The promoters of per, tim, vri, and Pdp1 were fully activated by CLK and strongly suppressed by CWO (Fig. 5A). Although we cannot exclude the possibility that overexpression might reveal molecular interactions that are not significant at physiological concentrations, this suppression effect is also observed in vivo and clearly correlated with the activity of CWO as the early morning troughs of per, tim, $v r i$, and $P d p 1$ mRNA at ZT3 are significantly elevated in cwo RNAi transgenic flies relative to those of wild-type flies (Fig. 5B). To further investigate how cwo contributes to the clock system in vivo, we measured the temporal expression levels of per, tim, vri, and Pdp1 in $c w O$ RNAi transgenic flies. The amplitude of expression of these genes decreased to half the level found in wild-type flies under LD conditions (Fig. 5C). The decreased amplitude of circadian oscillators in cwo RNAi transgenic flies relative to wild-type flies has been also confirmed under DD conditions (data not shown), suggesting that cwo functions to produce a high-amplitude oscillation of clock genes' expression. Furthermore, we also found that cwo strongly suppresses its own promoter (Fig. 5D). A similar effect was observed when we used the E-box2-luc construct as a suppression target. These results are consistent with the observation that trough levels of $c w O$ mRNA expression are elevated in cwo knockdown flies (Fig. 5E), which express reduced levels of CWO protein (Supplementary Fig. 2), and S2 cell experiments demonstrating that RNAi-mediated knockdown of cwo drastically reduces CWO levels without reducing cwo mRNA levels (Supplementary Fig. 5). Based on these results, we propose that cwo negatively regulates the expression of clock genes containing canonical circadian E-boxes, including $c$ wo itself, to form an autonegative feedback loop that functions to produce high-amplitude circadian transcription in Drosophila (Fig. 6).

\section{Discussion}

In this study, we adopted in vivo functional genomics strategy to identify a new core component in Drosophila clock. Our genome-wide screening using RNAi induced by a Gal4-UAS binary system has several advantages over previous clock mutant screenings and overcomes some of their limitations (Konopka and Benzer 1971; Blau and Young 1999; Martinek et al. 2001). Our method has proven to be one of the most concise and fastest ways to directly investigate how target genes function in the clock system. Importantly, targeting of RNAi expression using tissue-specific Gal4-driver lines allows us to avoid inducing lethality when we knock down essential genes for viability and development. Gene knockdowns of at least three new candidates including cwo induced lethality when we constitutively and ubiquitously expressed the dsRNA of the target gene (data not shown).

The identified clock gene $c w o$ is a transcriptional repressor and exhibits oscillatory expression under LD and DD, although the amplitude of this cwo mRNA oscillation in whole fly head is relatively modest (approximately twofold) compared with other transcriptional repressors such as per, tim, and vri. This may reflect a wider distribution of CWO protein within fly heads compared with PER protein since CWO is detected in additional cells within the fly brain (Fig. 2C). Alternatively, CWO function within the circadian oscillator may not require rhythms in the levels of CWO protein, precluding the need for high-amplitude cwo mRNA cycling. Importantly, cwo expression levels consistently reflect the levels of CLK-CYC activity, as shown in per $^{01}$ and Clk $^{\text {Irk }}$ mutants (Fig. 3A; Supplementary Fig. 4). Hence, the antagonistic competition between the cyclic CLK-CYC activity and the adjustable threshold imposed by CWO protein can be expected to keep E-box-mediated transcription within the controllable range of its activity, therefore rendering it more robust in generating highamplitude oscillation.

Consistent with this hypothesis, impaired activity of CWO leads to an elevated trough of per, tim, vri, and Pdp1mRNA at ZT3 in cwo RNAi transgenic flies compared with those of wild-type flies (Fig. 5C). As the fly circadian clock consists of complexly integrated negative and positive feedback loops (Fig. 6), this possible direct effect of impaired CWO activity may propagate and induce the indirect effects in the fly clock system. The observed lower peak level of per, tim, vri, and $P d p 1$ RNA expression in cwo RNAi transgenic flies (Fig. 5B) might reflect the indirect effects of impaired CWO activity. It is noteworthy that similar indirect effects on the oscillatory expression of per were observed in the hypomorphic tim ${ }^{\text {rit }}$ mutant, which exhibited a decreased activity of transcriptional repression by PER-TIM as 
A
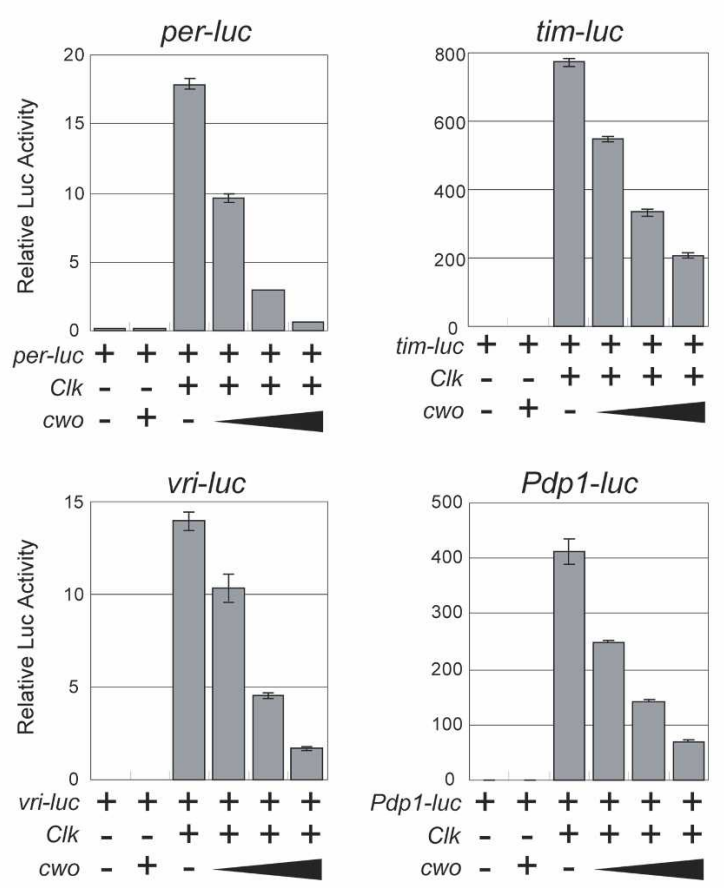

B

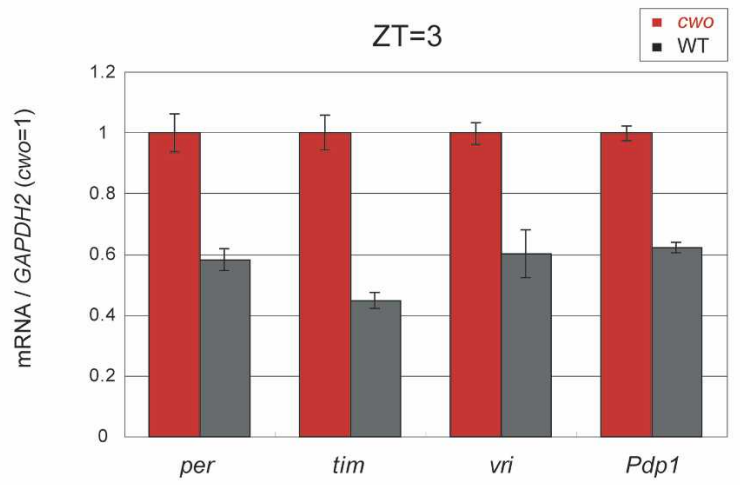

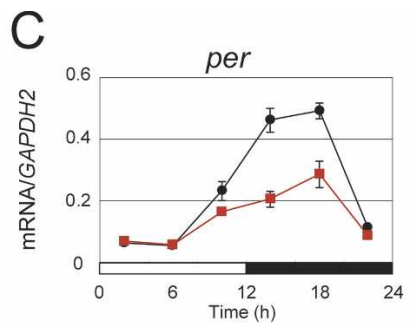

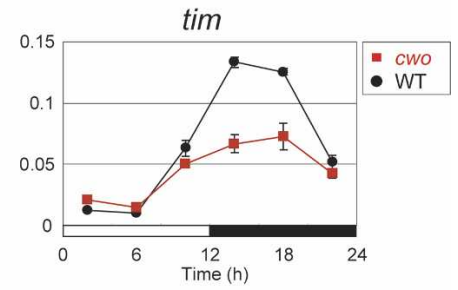

vri
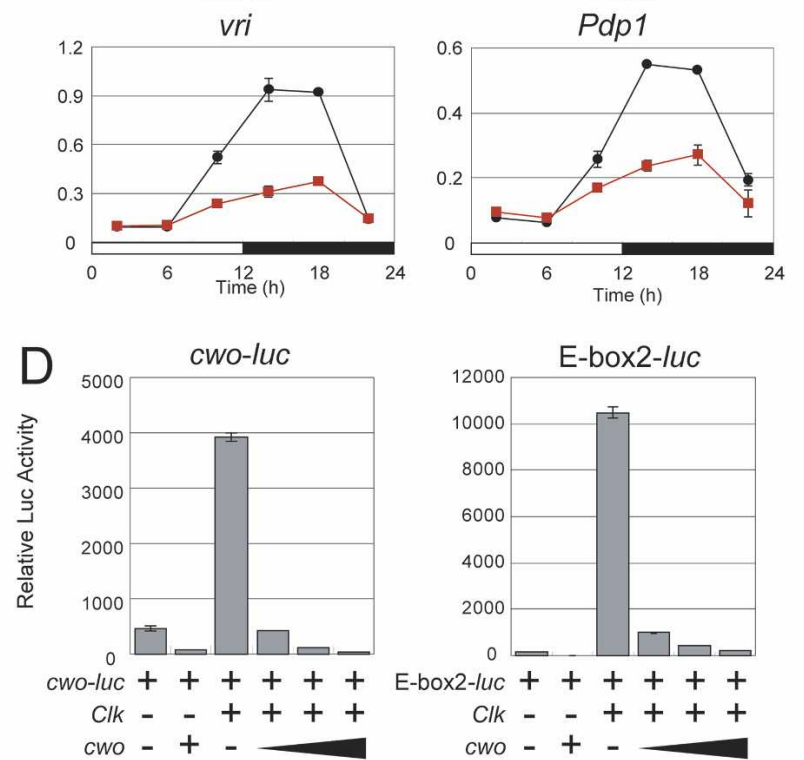

$E$

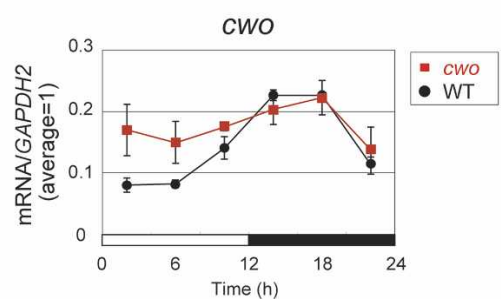

Figure 5. CWO protein regulates transcription of known clock genes. $(A)$ CWO protein suppresses transcription of known clock genes. Relative luciferase activities of per-luc, tim-luc, vri-luc, and Pdp1-luc in the presence of 0 and 100 ng of Clk and 0, 100, 400, and $800 \mathrm{ng}$ of $c w O$ are represented. Signals were normalized with Rluc activity. Error bars represent the SEM $(n=3)$. $(B)$ Expression of per, tim, vri, and Pdp1 mRNA at the trough level (ZT3) in wild-type (black) and cwo RNAi (red) flies under LD. Relative mRNA levels of each genes were measured with Q-PCR assay. GAPDH2 was used as an internal control. Data were normalized so that the average copy number in cwo RNAi flies is 1.0. Error bars represent the SEM $(n=3)$. $(C)$ Temporal expression profiles of per, tim, vri, and Pdp1 mRNA in wild-type (black circle) and cwo RNAi transgenic (red rectangle) flies under LD. The relative mRNA levels of each gene were measured with a Q-PCR assay. GAPDH2 was used as an internal control. Error bars represent the SEM $(n=2)$. We note that sampling time points (ZT1, ZT5, ZT9, ZT13, ZT17, and ZT21) of temporal expression profile are different from ZT3 in $B$. The expected trough phase calculated from these temporal expression data is almost ZT3, suggesting that the two data are consistent with each other. Since ZT1 and ZT5 are not exactly the trough phase, expression levels of clock genes at these timings are equal or slightly higher in wild-type flies than those of $c w o$ RNAi flies. (D) CWO protein suppresses its own transcription. Relative luciferase activities of $c w o-l u c$, and E-box2-luc in the presence of 0 and $100 \mathrm{ng}$ of Clk and 0,100,400, and $800 \mathrm{ng}$ of cwo are represented. Signals were normalized with Rluc activity. Error bars represent the SEM $(n=3)$. (E) Temporal expression profiles of $c w O$ mRNA in wild-type (black circle) and $c w O$ RNAi transgenic (red rectangle) flies under DD. Relative mRNA levels of each gene were measured with a Q-PCR assay. GAPDH2 was used as an internal control. Error bars represent the $\operatorname{SEM}(n=2)$.

well as the decreased peak level of per expression (Matsumoto et al. 1999|. Interestingly, the circadian phenotypes of this mutant are very similar to cwo knockdown flies showing $\sim 26 \mathrm{~h}$ in period.

Isolation of cwo as a new clock component and subsequent identification of a new negative feedback loop in this study revealed that transcriptional regulation through E-boxes is more complex than previously thought. The indirect autoregulatory negative feedback mechanism by PER and TIM through E-boxes, which is one of the key factors in circadian oscillation, has been extensively studied (Hardin 2005). This involves forma- 


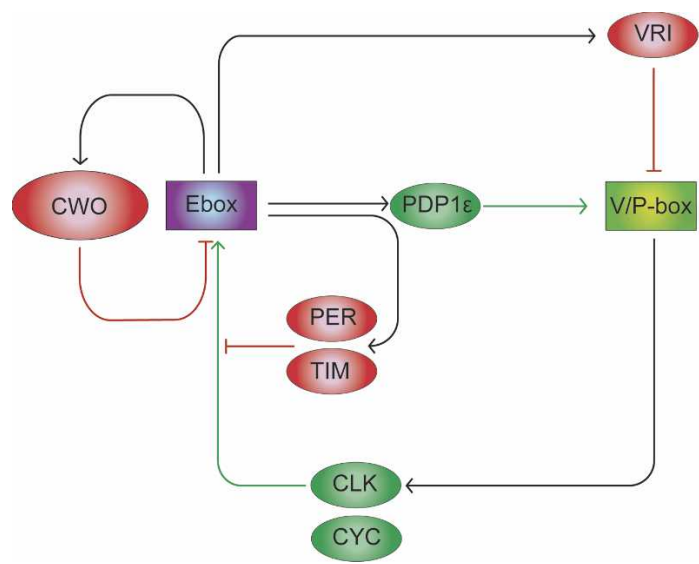

Figure 6. Transcriptional circuit underlying Drosophila circadian clock. Ellipsoids represent clock proteins, and rectangles represent time-of-day-specific DNA elements. Activators and repressors are represented in green and red, respectively. CWO protein directly binds to E-boxes and functions as a repressor.

tion of a heterodimer between PER and TIM, both of which lack a DNA-binding domain, that binds CLKCYC to inhibit the DNA-binding activity of CLK-CYC (Lee et al. 1999; Yu et al. 2006). The direct suppression mechanism through the E-box, however, had yet to be elucidated prior to this study. It has long been a mystery as to how the constitutive expression of per and tim in per $^{01}$; tim ${ }^{01}$ double mutants can rescue rhythmicity at the behavioral and molecular levels (Yang and Sehgal 2001). Our finding that $c w O$, one of the CLK-CYC target genes, can suppress the expression of a group of clock genes through binding to E-boxes suggests a new pathway for the negative feedback regulation in Drosophila clock. This negative regulation directly affects E-boxmediated transcription, contributing to sustaining a high-amplitude circadian expression, and may compensate the molecular rhythmicity even when a functional disorder occurs in other feedback loops.

\section{Materials and methods}

Genome-wide screening for rhythmically expressed genes

To extract the genes rhythmically expressed under both LD and DD in fly heads, we used two statistical cosine filters-one for the LD data set and the other for the DD data set obtained in a previous report (Ueda et al. 2002). We first calculated the best cross-correlation between 12-point time courses under LD of each probe set and cosine waves of defined periods and phases. For this, we prepared cosine waves of nine test periodicities from 20 to $28 \mathrm{~h}$ in increments of $1 \mathrm{~h}$. Cosine waves for each test period were considered over a total of 60 phases (i.e., peaks at 60 equally spaced times during the defined period), yielding a total of 540 test cosine waves. We performed the same procedure for the time courses under DD. Finally, we sorted all the probe sets by the sum of best correlations under LD and DD in a descending manner before selecting the top 200 probe sets for subsequent analysis.

\section{Measuring locomotor activity of flies}

Flies were kept under LD12:12 at $25^{\circ} \mathrm{C}$ and fed on standard glucose-cornmeal medium. Locomotor activity rhythm was re- corded and analyzed as described elsewhere (Matsumoto et al. 1999). The $w^{1118}$ strain was used as wild type. Knockdown flies were obtained by mating the Gal4 driver line to each of the UAS-IR lines.

Transgenic flies carrying a dominant-negative type of CWO protein

cwo cDNA lacking the "basic" region (cwo $\Delta \mathrm{b})$ of the bHLH domain was obtained by a PCR-based method using $\Delta$ b-forward and $\Delta \mathrm{b}$-reverse $5^{\prime}$-phospholylated primer sets with the cwo cDNAs cloned into a pIB/V5-His-TOPO plasmid (Invitrogen) as a template. Then a $c w o \Delta b$ BamHI fragment was cloned into the BglII site of the pUAS-T vector. The UAS-cwo $\Delta \mathrm{b}$ dominantnegative flies were obtained by $P$-element-mediated transformation. We established four UAS- $\Delta \mathrm{b}$ lines, each of which carries the transposon insertion at different sites of genome. Using $\Delta b-1$ and $\Delta \mathrm{b}-4$ lines, both of which have the insertion on the third chromosome, pdf-gal4; UAS-cwo $\Delta \mathrm{b}$ flies were established and recorded for their locomotor activities.

\section{Controls of genome-wide knockdown functional screening} in vivo

The gal4 driver lines used in this study have been reported to show no rhythm abnormalities (Blau and Young 1999; Renn et al. 1999; Malpel et al. 2002). The behavior of driverless IR lines for 20 different target genes ( 15\% of target genes) were recorded as a small-scale pilot study and displayed no rhythm abnormalities, which indicates that the IR construct alone does not cause rhythm abnormalities. Independent lines for each transgene with clock cell drivers were recorded in the full screening and show similar results. The possibility that each insert in independent lines for the same transgene would give similar results makes it unlikely that the phenotype is due to the insert alone. The genetic background of the knockdown lines $\left(w^{1118}\right)$ displayed normal rhythms as shown in Table 1.

Primer sequences for cwo $\Delta b$

$\Delta$ b-forward, 5'-GATCGCATGAACTCCTGCCTGGCGGACC TG-3'; $\Delta$ b-reverse, $5^{\prime}$-TCCGGTGGCATATTCAGCATCGTC CTCGCT-3'.

\section{CWO antibody production and immunolocalization}

Sequences coding for the CWO ORF were inserted into the pET28a vector as an NcoI/HindIII fragment, creating a fusion protein with a HIS-tag at the $\mathrm{C}$ terminus. The $\mathrm{NcoI}$ site contains the CWO translation start codon. The HindIII site was inserted 8 bp upstream of the natural translation stop codon by PCRmediated mutagenesis (primer sequence, CCGAAGCTTC CAGCTCTGGTTCATGGCC). CWO protein expression was induced in BL21 cells using isopropyl $\beta$-D-1-thiogalactopyranoside (IPTG). The cells were lysed and sonicated in $6 \mathrm{M}$ guanidium hydrochloride ( $\mathrm{pH}$ 8.0). The cell lysate was filtered through a $0.45-\mu \mathrm{m}$ filter and passed over a HiTrap Chelating HP column (Amersham Biosciences) charged with copper sulfate. Purified protein was eluted with increasing concentrations of imidazole. Pooled protein fractions were dialyzed against 0.05 M ammonium carbonate, frozen, and lyophilized. Purified CWO was resuspended in $6 \mathrm{M}$ urea and used to immunize guinea pigs for antibody production (Cocalico Biological).

Immunofluorescent detection of CWO and PER in brains from wild-type and homozygous f05073 mutant flies collected at ZT21 was carried out as described (Houl et al. 2006). CWO antibody (GP27) was diluted 1:1000, and rabbit PER antibody preabsorbed against per ${ }^{01}$ embryos was diluted 1:15,000. Alexa Fluor 488-conjugated anti-guinea pig and Alexa Fluor 568-con- 
jugated anti-rabbit secondary antibodies (Molecular Probes/Invitrogen) were diluted to a final concentration of 1:200. Brains were optically scanned at a thickness of $2 \mu \mathrm{m}$ using an Olympus FV1000 confocal microscope, and images were processed using Adobe Photoshop. Images are representative of 12 brain hemispheres from wild-type and 12 brain hemispheres from f05073 mutants.

\section{Q-PCR of temporal expression level}

Q-PCR was performed using ABI Prism 7700 and SYBR Green Reagents (Applied Biosystems). Wild-type flies were sampled every $4 \mathrm{~h}$ in LD and DD for $2 \mathrm{~d}(n=2)$. tim(UAS)-gal4; cwo-IR2 RNAi transgenic flies were sampled every $4 \mathrm{~h}$ in $\operatorname{LD}(n=2)$. per $^{01}$ and Clk ${ }^{r k k}$ mutants were sampled every $6 \mathrm{~h}$ in $\mathrm{LD}(n=2)$. Total RNA was purified from $\sim 100$ heads at each time point as described elsewhere (Ueda et al. 2002). cDNA was synthesized from $0.5 \mu \mathrm{g}$ of total RNA using random 6-mer (Promega) and SuperScript II Reverse Transcriptase (Invitrogen) according to the standard protocol. The amplified DNA sample was measured for verification of the ChIP experiment. Samples contained $1 \times$ SYBR Green Master Mix (Applied Biosystems), $0.5 \mu \mathrm{M}$ primers, and $1 / 40$ synthesized cDNA in a $25-\mu \mathrm{L}$ volume. The PCR conditions were as follows: $10 \mathrm{~min}$ at $95^{\circ} \mathrm{C}$, then 40 cycles of $15 \mathrm{sec}$ at $94^{\circ} \mathrm{C}, 30 \mathrm{sec}$ at $59^{\circ} \mathrm{C}$, and $1 \mathrm{~min}$ at $72^{\circ} \mathrm{C}$. Absolute cDNA abundance was calculated using the standard curve obtained from Drosophila genomic DNA. GAPDH2 expression levels were quantified and used as the internal control.

Primer sequences for Q-PCR of Drosophila $c D N A$

cwo-forward, 5'-ATCTGCGCCCAAGTGTACCT-3'; cwo-reverse, 5'-TGCTTCTCCTCCATTTCCATTAAC-3'; per-forward, 5'-ATAAGCACAACGACGAGATGGA-3'; per-reverse, 5'-GAGCCTCCTCTTTTTATCCCGT-3'; tim-forward, 5'-CA CACCATCTTCGAGCTGAATAA-3'; tim-reverse, 5'-AGTT GTTGATTTGATCGCATTTG-3'; $P d p 1 \varepsilon$-forward, 5'-GCAAC TGGTAATGGAAATGGTG-3'; $P d p 1 \varepsilon$-reverse, 5 '-CTGTTC AAATGGTTGTGATGCTC-3'; vri-forward, 5'-CATCACTAC AGCCAGCAGAAGC-3'; vri-reverse, 5' -ATATTGGATAGCC GGACGTTGT-3'; GAPDH2-forward, 5'-CTACCTGTTCAA GTTCGATTCGAC-3'; GAPDH2-reverse, 5'-AGTGGACTC CACGATGTATTCG-3'

\section{Primer sequences for Q-PCR of mouse cDNA}

Hes5-forward, 5'-TCTCCACGATGATCCTTAAAGGA-3'; Hes5reverse, 5'-CAAAATCGTGCCCACATGC-3'; Tbp-forward, 5'GTTGTGCAGAAGTTGGGCTTC-3'; Tbp-reverse, 5'-TCACA GCTCCCCACCATGTT-3'.

\section{Primer sequences for Q-PCR of ChIP products}

cwo-5'-forward, 5'-GAGTAACCCGAGCCCGTTTT-3'; cwo5'-reverse, 5'-ATACGACCGGTTGGTTGCTT-3'; Pdp1-5'\#1forward, 5'-GGATTGGTTCCCTATTCTCGG-3'; Pdp1-5'\#1reverse, 5'-GCAGAGCTTTGCTATTGGCC-3'; $P d p 1$ - $5^{\prime}$ '\#2-forward, 5'-TCTTGCGTCGGCACTTGAG-3'; Pdp1-5'\#2-reverse, 5'-TTATCGAGCACCCCAACATTT-3'; vrille-5'-forward, 5' TCGCAATCGAAATCGGACTC-3'; vrille-5'-reverse, 5'-ACG CACACACACGTAAGGTGAC-3'; Act75B-5'-forward, 5'-GA TACGATCTCCACGCTCGATC-3'; Act75B-5'-reverse, 5'-GG AATACCATATAGCATATAGCGCAG-3' .

\section{Transfection and luciferase assay}

A luciferase reporter plasmid was constructed based on a pGL3basic vector (Promega). For Figure 5, A and B, each promoter region of $\operatorname{vri}(2.5 \mathrm{~kb}), P d p 1(4.1 \mathrm{~kb})$, and $c w o(2.75 \mathrm{~kb})$ was amplified by PCR from a $w^{1118}$ fly genome and subcloned into a pCRII vector (Invitrogen). The XbaI-SpeI fragment of vri or the $P d p 1$ promoter region was then cloned into the NheI site of the pGL3-basic vector, while the HindIII fragment of the cwo promoter region was cloned into the HindIII site of the pGL3-basic vector. For Figure 3C, oligonucleotides (Hokkaido System Science) containing three tandem repeats of wild-type or mutant E-boxes from the cwo promoter region were annealed and inserted into the HindIII site upstream of TATA-Luc, which contains a minimal promoter region of the $h s p 70$ gene at the HindIII-NcoI site of pGL3-basic. A pAc5.1-Rluc reporter was also constructed by inserting the Rluc (Renilla luciferase) gene of the pRL-CMV vector (Promega) into pAc5.1B (Invitrogen).

Drosophila S2 cells were maintained in Schneider's Drosophila medium (Invitrogen) supplemented with 10\% FBS (Bioserum) and antibiotics (12.5 U/mL penicillin, $12.5 \mathrm{mg} / \mathrm{mL}$ streptomycin; GIBCO). S2 cells were plated onto six-well plates at $4 \times 10^{6}$ cells per well density the day before transfection. The following day, S2 cells were transfected using Effectene Transfection Reagent (Qiagen) with $100 \mathrm{ng}$ of the promoter reporter construct in the presence of 0 and $100 \mathrm{ng}$ of pAct-Clk (Darlington et al. 1998), and 0, 100, 400, and 800 ng of pAc5.1-Flag-cwo by a standard transfection method. The total amount of transfected plasmid was equaled to $1.1 \mathrm{\mu g}$ by pAc5.1B empty vector (Invitrogen). Additionally, $5 \mathrm{ng}$ of an Rluc reporter vector, pAc5.1-RLuc, were added into each transfection as an internal control for transfection efficiency. Forty-eight hours after transfection, transfected cells were harvested and assayed with the Dual-Luciferase Reporter Assay System (Promega). Luciferase activity was normalized by Rluc activity.

Primer sequences for amplifications of vri, Pdp1, and cwo promoter region

vriG3564-forward, 5' -GCTAGGGAACAAACGCAATAAGTG3'; vriG6085-reverse, 5'-TCGAAAGAAGGCTGAATCGCAA3'; Pdp1G30257-forward, 5'-CATACACAGGCACAAGACC GTT-3'; Pdp1G35327-reverse, 5'-TTCGTGTGGTCAGGGA ATGCAC-3'; cwoG0973-forward, 5'-TAAGCTTAGCCATG CAATCGGGGTAAACAG-3'; cwoG3681-reverse, 5'-TAAGC TATGTACGGGCGAGCGAGCAACGTG-3'.

\section{Oligonucleotide sequences}

E-boxl-forward, 5'-CGCGACGTTTCACGTGTTTTATACGT TTCACGTGTTTTATACGTTTCACGTGTTTTAT-3'; E-box1reverse, 5'-GATCATAAAACACGTGAAACGTATAAAACACG TGAAACGTATAAAACACGTGAAACGT-3'; E-box2-forward, 5'-CGCGCAACAACACGTGACCAACCAACAACACGTGAC CAACCAACAACACGTGACCAAC-3'; E-box2-reverse, 5'-GAT CGTTGGTCACGTGTTGTTGGTTGGTCACGTGTTGTTGG TTGGTCACGTGTTGTTG-3'; E-box1-mutant-forward, 5'-CGC GACGTTTACCGGTTTTTATACGTTTACCGGTTTTTATAC GTTTACCGGTTTTTAT-3'; E-boxl-mutant-reverse, 5'-GAT CATAAAAACCGGTAAACGTATAAAAACCGGTAAACGTA TAAAAACCGGTAAACGT-3'; E-box2-mutant-forward, 5'-CG CGCAACAAACCGGTACCAACCAACAAACCGGTACCAAC CAACAAACCGGTACCAAC-3'; E-box2-mutant-reverse, 5'-GA TCGTTGGTACCGGTTTGTTGGTTGGTACCGGTTTGTTG GTTGGTACCGGTTTGTTG-3'.

\section{Construction of cwo expression vector}

To construct the Flag-tagged cwo expression vector, a pMT/V5His-B plasmid (Invitrogen) was digested with EcoRV and XhoI and blunt-ended with T4 DNA polymerase. An HU2 cassette 
containing a Kozak sequence, start codon, Flag tag sequence, I-SceI recognition sequence, PI-PspI recognition sequence, and stop codon (all of them in-frame) was amplified from a pMU2 plasmid (H. Ukai, T.J. Kobayashi, M. Nagano, K. Masumoto, M. Sujino, T. Kondo, K. Yagita, Y. Shigeyoshi, and H.R. Ueda, in prep.) by PCR and inserted into the digested sites of pMT/V5His-B and termed pMT-Flag. The full coding sequence of $c$ wo was amplified by PCR from the head cDNA of a $w^{1118}$ fly with a forward primer containing an I-SceI recognition sequence and a reverse primer containing a PI-PspI recognition sequence. PCR products were digested with I-SceI and PI-PspI and inserted into a pMT-Flag vector and termed pMT-Flag-cwo.

\section{Primer sequences for HU2 cassette amplification}

HU2-forward, 5'-GTGACACTATAGAATACAAGC-3'; HU2reverse, 5'-CCGCACCCGACATAGATTC-3'.

\section{Primer sequences for ORF amplification}

cwo-forward, 5'-ATTACCCTGTTATCCCTAATCCGCCCAT CCGGTCAAG-3'; cwo-reverse, 5' -ACCCATAATACCCATAA TAGCTGTTTGCCACCAGCTCTGGTTCATGGC-3'.

\section{ChIP assay with Drosophila genome tiling chip}

S2 cells were transfected with pMT-Flag-cwo $(1.9 \mu \mathrm{g})$ or pMTFlag $(1.9 \mu \mathrm{g})$ and pCoHygro $(0.1 \mu \mathrm{g}$; Invitrogen, for selection) using Effectene (Qiagen) according to the manufacturer's instructions. Hygromycin-B $(300 \mu \mathrm{g} / \mathrm{mL}$ final concentration; Invitrogen) was added $5 \mathrm{~d}$ after transfection. After $3 \mathrm{wk}$, the established stable cell lines were used for the ChIP assay.

Cells $\left(4 \times 10^{7}\right)$ were collected and stimulated by copper sulfate to induce fusion genes. Cells were harvested $24 \mathrm{~h}$ after stimulation. ChIP was then performed according to the previously described method (Yamashita et al. 2002) with some modifications. In this report, we used Chromatin Immunoprecipitation Assay Kits (Upstate Biotechnology), anti-Flag M2 antibody (Sigma), and anti-V5 antibody (Sigma). Protein G-Sepharose (Amersham) was used for precipitation of antibody/protein immune complexes. The resulting precipitated DNA was amplified by adaptor-mediated PCR (Cawley et al. 2004) and then fragmented to $50-150$ bp by DNase I (Epicentre) in One-Phor-All buffer (Pharmacia). The distribution of fragmented DNA size was verified on a $2 \%$ agarose gel. The fragmented DNA was then end-labeled with GeneChip Labeling Reagent (Affymetrix) by using terminal deoxynucleotidyltransferase (Roche) in TdT buffer (Roche) and $\mathrm{CoCl}_{2}$ (Roche). To prepare the hybridization cocktail, $5 \mu \mathrm{g}$ of labeled DNA fragment were mixed with final $1 \times$ MES, 3 M TMAC, $0.02 \%$ Triton (Sigma), 50 pM Oligo B2, 1× Hybridization Control (Affymetrix), and $100 \mu \mathrm{g} / \mu \mathrm{L}$ Herring Sperm DNA (Promega). Two-hundred microliters of Hybridization cocktail were applied to a Drosophila genome tiling array (Affymetrix) and hybridized for $18 \mathrm{~h}$ at $45^{\circ} \mathrm{C}$ with $45 \mathrm{rpm}$ rotation. Stain and wash was executed by a Fluidics station 450 with the EukGE-WS2 version 4 program. Arrays were scanned by a GeneChip Scanner 7G (Affymetrix).

\section{Western blot analysis of CWO in cwo RNAi fly}

Fly heads were homogenized with a pestle, and protein extracts were prepared with NE-PER Nuclear and Cytoplasmic Extraction Reagents (Pierce). The extracts of 40 heads were applied to one lane of a polyacrylamide gel. For immunoblot analysis, proteins were transferred to PVDF membranes (Invitrogen) and incubated with diluted antibodies in blocking solution. Anti-
CWO antiserum (GP25) was diluted 2000-fold, Horseradish Peroxidase (HRP)-conjugated anti-guinea pig secondary antibody (ICN) was diluted 1000-fold, anti-Tubulin-antibody (DM1A, Lab Vision) was diluted 300-fold, and HRP-conjugated antimouse secondary antibody (Amersham) was diluted 2000-fold. For visualization, the ECL Plus Western Blotting Detection System (Amersham) and LAS-3000mini (FujiFilm) were used according to the manufacturers' instructions.

\section{Western blot analysis of CWO protein and Q-PCR of cwo mRNA}

Drosophila S2 cells were plated onto six-well plates at $4 \times 10^{6}$ cells per well density the day before transfection. The following day, S2 cells were transfected using Effectene Transfection Reagent (Qiagen) with $500 \mathrm{ng}$ of pMT-Flag-cwo, $200 \mathrm{ng}$ of pWAGAL4 (a kind gift of Y. Hiromi), $300 \mathrm{ng}$ of pUAS-T empty plasmid, and pUAS-cwo-IR by a standard transfection method. Additionally, $20 \mathrm{ng}$ of pMT-Flag-GFP were added into each transfection as an internal control for transfection efficiency. A total amount of transfected plasmid was equaled to $1.1 \mu \mathrm{g}$ by pAc5.1B empty vector (Invitrogen). Forty-eight hours after transfection, the recombinant proteins' expression was induced with $500 \mu \mathrm{M}$ copper sulfate. Twenty-four hours after the induction, transfected cells were harvested and used for Western blot analysis and Q-PCR.

For Western blot analysis, each nuclear and cytoplasmic fraction was extracted from the harvested cells with NE-PER Nuclear and Cytoplasmic Extraction Reagents (Pierce), and each cell lysate of $4 \times 10^{6}$ cells was applied to a lane of polyacrylamide gel. For immunoblot analysis, proteins were transferred to PVDF membranes. Anti-Flag M2 monoclonal Antibody-Peroxidase Conjugate (Sigma) was diluted 2000-fold and used for detection. For visualization, the ECL Plus Western Blotting Detection System (Amersham) and LAS-3000mini (FujiFilm) were used according to the manufacturers' instructions.

Q-PCR was performed using ABI Prism 7700 and SYBR Green Reagents (Applied Biosystems). Total RNA was purified from the harvested cells using TRIzol reagent (Invitrogen). cDNA was synthesized from $0.25 \mu \mathrm{g}$ of total RNA with random 6-mer (Promega) and SuperScript II Reverse Transcriptase (Invitrogen) according to the standard protocol. Samples contained $1 \times$ SYBR Green Master Mix (Applied Biosystems), $0.5 \mu \mathrm{M}$ primers, and $1 / 20$ synthesized cDNA in a $25-\mu \mathrm{L}$ volume. The PCR conditions were as follows: $10 \mathrm{~min}$ at $95^{\circ} \mathrm{C}$, then 40 cycles of $15 \mathrm{sec}$ at $94^{\circ} \mathrm{C}, 30 \mathrm{sec}$ at $59^{\circ} \mathrm{C}$, and $1 \mathrm{~min}$ at $72^{\circ} \mathrm{C}$. Absolute cDNA abundance was calculated using the standard curve obtained from Drosophila genomic DNA, pMT-Flag-cwo, and pMT-Flag-GFP plasmid. GAPDH2 or Flag-GFP expression levels were quantified and used as the internal control.

\section{Primer sequences for $Q-P C R$}

cwo-forward, 5'-ATCTGCGCCCAAGTGTACCT-3'; cwo-reverse, 5'-TGCTTCTCСТCСАТTTCСАTTAAC-3'; GAPDH2forward, 5'-CTACCTGTTCAAGTTCGATTCGAC-3'; GAP DH2-reverse, 5'-AGTGGACTCCACGATGTATTCG-3'; Flagforward, 5'-GACTACAAGGATGACGATGAC-3'; cwo-reverse2, 5'-ACTGGAGACAGCTCTCATAC-3'; GFP-forward, 5' - CCATGGCCAACACTTGTCAC-3'; GFP-reverse, 5' - TCC GGATAACGGGAAAAGC-3'

\section{Tiling array data analysis}

For the identification of transcription factor-binding sites (TFBSs) of $c$ wo, we used data obtained from four arrays: two for 
Flag-antibody and two for V5-antibody. The four sets of array data were analyzed by TileMap (Ji and Wong 2005). In the resulting BED file, we selected TFBSs with a score of 999 (the highest score) for further analysis to achieve high stringency. Detailed information for these detected TFBSs is available online at https://alcyone.cdb.riken.jp/papers/cwo. Array data including raw data are available at Gene Expression Omnibus (http://www.ncbi.nlm.nih.gov/geo) via series accession number GSE7569. To find the binding motif of cwo, we first selected TFBSs within $2 \mathrm{~kb}$ of the transcription start sites of known genes. We then inputted the corresponding genomic sequences to Weeder (Pavesi et al. 2004) running on a local computer. The sequence logo in Figure 4D was drawn by EnoLOGOS (Workman et al. 2005).

\section{Statistical significance of E-box frequencies in cwo promoter}

To assess the statistical significance of observing the canonical E-box, E'-box, and noncanonical E-box frequencies in the $\sim 2.8$ $\mathrm{kb}$ promoter region of $c w 0$ ( 2550 bp upstream of and $\sim 250 \mathrm{bp}$ downstream from the TSS), we generated 10,000 random sequences with the length of $2.8 \mathrm{~kb}$, then counted occurrences of canonical E-boxes, E'-boxes, and noncanonical E-boxes in those sequences, respectively. The random sequences were generated so that the dinucleotide frequency was same as that of promoter regions in the Drosophila genome. Nonoverlapping 7021 promoter regions were sampled and used for calculating dinucleotide frequency. For the analysis of the first intron, 11,637 nonoverlapping first intron regions were sampled and used for calculating dinucleotide frequency. Then we generated 10,000 random sequences with a length of $7.3 \mathrm{~kb}$.

\section{Acknowledgments}

We thank Kenji Tomioka, Taishi Yoshii, Miki Shimohigashi, Ayami Matsushima, and Yasuyuki Shimohigashi for useful discussions; Yasushi Hiromi for providing pWAGAL4; and Steve A. Kay for providing pAct-Clk, per-luc, and tim-luc plasmids. The tim(UAS)-gal4 strain was a kind gift of Justin Blau; pdf-gal4 was donated by Jeffrey C. Hall; gal $^{1118}$ was donated by Francois Rouyer; and a cwo-deficient strain, f05073, was donated by the Exelixis collection, Harvard University. We thank Ueli Schibler for critical reading of this manuscript and also thank Michael Rosbash and Ravi Allada for sharing information before publication. We thank Kiyo Kimura, Makiko Haruta, Kazuko Fujitani, and Yukoko Sado for technical assistance and Douglas Sipp and Michael Royle for proofreading of the manuscript. We thank Philipp Kapranov, Srinka Ghosh, Michael Paumen, and Tom R. Gingeras for technical support on both the ChIP assay and data analysis with a Drosophila genome tiling chip. This work is supported in part by an intramural Grant-in-Aid from CDB (to H.R.U.); the President's Fund from RIKEN (to H.R.U.); a Grant-in-Aid for Scientific Research from the Ministry of Education, Culture, Sports, Science and Technology of Japan (no. 17570134 and no. 18016025 to A.M., no. 17017032 and National BioResource Project to R.U., and Systems Genomics Project to H.R.U.); a Grant-in-Aid from the Uehara Memorial Foundation (to H.R.U.); and an NIH grant NS052854 (to P.E.H.).

\section{References}

Allada, R., White, N.E., So, W.V., Hall, J.C., and Rosbash, M. 1998. A mutant Drosophila homolog of mammalian Clock disrupts circadian rhythms and transcription of period and timeless. Cell 93: 791-804.
Blau, J. and Young, M.W. 1999. Cycling vrille expression is required for a functional Drosophila clock. Cell 99: 661-671.

Cawley, S., Bekiranov, S., Ng, H.H., Kapranov, P., Sekinger, E.A., Kampa, D., Piccolboni, A., Sementchenko, V., Cheng, J., Williams, A.J., et al. 2004. Unbiased mapping of transcription factor binding sites along human chromosomes 21 and 22 points to widespread regulation of noncoding RNAs. Cell 116: 499-509.

Ceriani, M.F., Hogenesch, J.B., Yanovsky, M., Panda, S., Straume, M., and Kay, S.A. 2002. Genome-wide expression analysis in Drosophila reveals genes controlling circadian behavior. J. Neurosci. 22: 9305-9319.

Claridge-Chang, A., Wijnen, H., Naef, F., Boothroyd, C., Rajewsky, N., and Young, M.W. 2001. Circadian regulation of gene expression systems in the Drosophila head. Neuron 32: 657671.

Curtin, K.D., Huang, Z.J., and Rosbash, M. 1995. Temporally regulated nuclear entry of the Drosophila period protein contributes to the circadian clock. Neuron 14: 365-372.

Darlington, T.K., Wager-Smith, K., Ceriani, M.F., Staknis, D., Gekakis, N., Steeves, T.D., Weitz, C.J., Takahashi, J.S., and Kay, S.A. 1998. Closing the circadian loop: CLOCK-induced transcription of its own inhibitors per and tim. Science 280: 1599-1603.

Darlington, T.K., Lyons, L.C., Hardin, P.E., and Kay, S.A. 2000. The period E-box is sufficient to drive circadian oscillation of transcription in vivo. J. Biol. Rhythms 15: 462-471.

Davis, R.L. and Turner, D.L. 2001. Vertebrate hairy and Enhancer of split related proteins: Transcriptional repressors regulating cellular differentiation and embryonic patterning. Oncogene 20: 8342-8357.

Dubruille, R., Laurencon, A., Vandaele, C., Shishido, E., Coulon-Bublex, M., Swoboda, P., Couble, P., Kernan, M., and Durand, B. 2002. Drosophila regulatory factor X is necessary for ciliated sensory neuron differentiation. Development 129: $5487-5498$.

Glossop, N.R., Houl, J.H., Zheng, H., Ng, F.S., Dudek, S.M., and Hardin, P.E. 2003. VRILLE feeds back to control circadian transcription of Clock in the Drosophila circadian oscillator. Neuron 37: 249-261.

Grechez-Cassiau, A., Panda, S., Lacoche, S., Teboul, M., Azmi, S., Laudet, V., Hogenesch, J.B., Taneja, R., and Delaunay, F. 2004. The transcriptional repressor STRA13 regulates a subset of peripheral circadian outputs. J. Biol. Chem. 279: 11411150.

Grima, B., Chelot, E., Xia, R., and Rouyer, F. 2004. Morning and evening peaks of activity rely on different clock neurons of the Drosophila brain. Nature 431: 869-873.

Hao, H., Glossop, N.R., Lyons, L., Qiu, J., Morrish, B., Cheng, Y., Helfrich-Forster, C., and Hardin, P. 1999. The 69 bp circadian regulatory sequence (CRS) mediates per-like developmental, spatial, and circadian expression and behavioral rescue in Drosophila. J. Neurosci. 19: 987-994.

Hardin, P.E. 2005. The circadian timekeeping system of Drosophila. Curr. Biol. 15: R714-R722.

Hinrichs, A.S., Karolchik, D., Baertsch, R., Barber, G.P., Bejerano, G., Clawson, H., Diekhans, M., Furey, T.S., Harte, R.A., Hsu, F., et al. 2006. The UCSC Genome Browser Database: Update 2006. Nucleic Acids Res. 34: D590-D598.

Honma, S., Kawamoto, T., Takagi, Y., Fujimoto, K., Sato, F., Noshiro, M., Kato, Y., and Honma, K. 2002. Dec1 and Dec2 are regulators of the mammalian molecular clock. Nature 419: 841-844.

Houl, J.H., Yu, W., Dudek, S.M., and Hardin, P.E. 2006. Drosophila CLOCK is constitutively expressed in circadian oscillator and non-oscillator cells. J. Biol. Rhythms 21: 93-103. 
Hunter-Ensor, M., Ousley, A., and Sehgal, A. 1996. Regulation of the Drosophila protein timeless suggests a mechanism for resetting the circadian clock by light. Cell 84: 677-685.

Ji, H. and Wong, W.H. 2005. TileMap: Create chromosomal map of tiling array hybridizations. Bioinformatics 21: 3629-3636.

Kadener, S., Stoleru, D., McDonald, M., Nawathean, P., and Rosbash, M. 2007. Clockwork Orange is a transcriptional repressor and a new Drosophila circadian pacemaker component. Genes \& Dev. (this issue), doi: 10.1101/gad.1552607.

Konopka, R.J. and Benzer, S. 1971. Clock mutants of Drosophila melanogaster. Proc. Nat1. Acad. Sci. 68: 2112-2116.

Lee, C., Bae, K., and Edery, I. 1999. PER and TIM inhibit the DNA binding activity of a Drosophila CLOCK-CYC/ dBMAL1 heterodimer without disrupting formation of the heterodimer: A basis for circadian transcription. Mol. Cell. Biol. 19: 5316-5325.

Lim, C., Chung, B.Y., Pitman, J.L., McGill, J.J. Pradhan, S., Lee, J., Keegan, K.P., Choe, J., and Allada, R. 2007. clockwork orange encodes a transcriptional repressor important for circadian clock amplitude in Drosophila. Curr. Biol. (in press).

Lin, Y., Han, M., Shimada, B., Wang, L., Gibler, T.M., Amarakone, A., Awad, T.A., Stormo, G.D., Van Gelder, R.N., and Taghert, P.H. 2002. Influence of the period-dependent circadian clock on diurnal, circadian, and aperiodic gene expression in Drosophila melanogaster. Proc. Nat1. Acad. Sci. 99: 9562-9567.

Lyons, L.C., Darlington, T.K., Hao, H., Houl, J., Kay, S.A., and Hardin, P.E. 2000. Specific sequences outside the E-box are required for proper per expression and behavioral rescue. $J$. Biol. Rhythms 15: 472-482.

Malpel, S., Klarsfeld, A., and Rouyer, F. 2002. Larval optic nerve and adult extra-retinal photoreceptors sequentially associate with clock neurons during Drosophila brain development. Development 129: 1443-1453.

Manak, J.R., Dike, S., Sementchenko, V., Kapranov, P., Biemar, F., Long, J., Cheng, J., Bell, I., Ghosh, S., Piccolboni, A., et al. 2006. Biological function of unannotated transcription during the early development of Drosophila melanogaster. Nat. Genet. 38: 1151-1158.

Martinek, S. and Young, M.W. 2000. Specific genetic interference with behavioral rhythms in Drosophila by expression of inverted repeats. Genetics 156: 1717-1725.

Martinek, S., Inonog, S., Manoukian, A.S., and Young, M.W. 2001. A role for the segment polarity gene shaggy/GSK-3 in the Drosophila circadian clock. Cell 105: 769-779.

Matsumoto, A., Tomioka, K., Chiba, Y., and Tanimura, T. 1999. tim $^{\text {rit }}$ lengthens circadian period in a temperature-dependent manner through suppression of PERIOD protein cycling and nuclear localization. Mol. Cell. Biol. 19: 4343-4354.

McDonald, M.J. and Rosbash, M. 2001. Microarray analysis and organization of circadian gene expression in Drosophila. Cell 107: $567-578$

McDonald, M.J., Rosbash, M., and Emery, P. 2001. Wild-type circadian rhythmicity is dependent on closely spaced $\mathrm{E}$ boxes in the Drosophila timeless promoter. Mol. Cell. Biol. 21: 1207-1217.

Naito, Y., Yamada, T., Matsumiya, T., Ui-Tei, K., Saigo, K., and Morishita, S. 2005. dsCheck: Highly sensitive off-target search software for double-stranded RNA-mediated RNA interference. Nucleic Acids Res. 33: W589-W591.

Pavesi, G., Mereghetti, P., Mauri, G., and Pesole, G. 2004. Weeder Web: Discovery of transcription factor binding sites in a set of sequences from co-regulated genes. Nucleic Acids Res. 32: W199-W203.

Pili-Floury, S., Leulier, F., Takahashi, K., Saigo, K., Samain, E., Ueda, R., and Lemaitre, B. 2004. In vivo RNA interference analysis reveals an unexpected role for GNBP1 in the defense against Gram-positive bacterial infection in Drosophila adults. J. Biol. Chem. 279: 12848-12853.

Price, J.L., Blau, J., Rothenfluh, A., Abodeely, M., Kloss, B., and Young, M.W. 1998. double-time is a novel Drosophila clock gene that regulates PERIOD protein accumulation. Cell 94: 83-95.

Renn, S.C., Park, J.H., Rosbash, M., Hall, J.C., and Taghert, P.H. 1999. A pdf neuropeptide gene mutation and ablation of PDF neurons each cause severe abnormalities of behavioral circadian rhythms in Drosophila. Cell 99: 791-802.

Rouyer, F., Rachidi, M., Pikielny, C., and Rosbash, M. 1997. A new gene encoding a putative transcription factor regulated by the Drosophila circadian clock. EMBO J. 16: 3944-3954.

Rutila, J.E., Suri, V., Le, M., So, W.V., Rosbash, M., and Hall, J.C. 1998. CYCLE is a second bHLH-PAS clock protein essential for circadian rhythmicity and transcription of Drosophila period and timeless. Cell 93: 805-814.

Sehgal, A., Price, J.L., Man, B., and Young, M.W. 1994. Loss of circadian behavioral rhythms and per RNA oscillations in the Drosophila mutant timeless. Science 263: 1603-1606.

Shafer, O.T., Helfrich-Forster, C., Renn, S.C., and Taghert, P.H. 2006. Reevaluation of Drosophila melanogaster's neuronal circadian pacemakers reveals new neuronal classes. J. Comp. Neurol. 498: 180-193.

Stempfl, T., Vogel, M., Szabo, G., Wulbeck, C., Liu, J., Hall, J.C., and Stanewsky, R. 2002. Identification of circadian-clockregulated enhancers and genes of Drosophila melanogaster by transposon mobilization and luciferase reporting of cyclical gene expression. Genetics 160: 571-593.

Ueda, H.R., Matsumoto, A., Kawamura, M., Iino, M., Tanimura, T., and Hashimoto, S. 2002. Genome-wide transcriptional orchestration of circadian rhythms in Drosophila. J. Biol. Chem. 277: 14048-14052.

Van Gelder, R.N. and Krasnow, M.A. 1996. A novel circadianly expressed Drosophila melanogaster gene dependent on the period gene for its rhythmic expression. EMBO J. 15: 16251631.

Van Gelder, R.N., Bae, H., Palazzolo, M.J., and Krasnow, M.A. 1995. Extent and character of circadian gene expression in Drosophila melanogaster: Identification of twenty oscillating mRNAs in the fly head. Curr. Biol. 5: 1424-1436.

Workman, C.T., Yin, Y., Corcoran, D.L., Ideker, T., Stormo, G.D., and Benos, P.V. 2005. enoLOGOS: A versatile Web tool for energy normalized sequence logos. Nucleic Acids Res. 33: W389-W392.

Yamashita, M., Ukai-Tadenuma, M., Kimura, M., Omori, M., Inami, M., Taniguchi, M., and Nakayama, T. 2002. Identification of a conserved GATA3 response element upstream proximal from the interleukin-13 gene locus. J. Biol. Chem. 277: 42399-42408.

Yang, Z. and Sehgal, A. 2001. Role of molecular oscillations in generating behavioral rhythms in Drosophila. Neuron 29: 453-467.

Yu, W., Zheng, H., Houl, J.H., Dauwalder, B., and Hardin, P.E. 2006. PER-dependent rhythms in CLK phosphorylation and E-box binding regulate circadian transcription. Genes \& Dev. 20: 723-733.

Zerr, D.M., Hall, J.C., Rosbash, M., and Siwicki, K.K. 1990. Circadian fluctuations of period protein immunoreactivity in the CNS and the visual system of Drosophila. J. Neurosci. 10: $2749-2762$. 


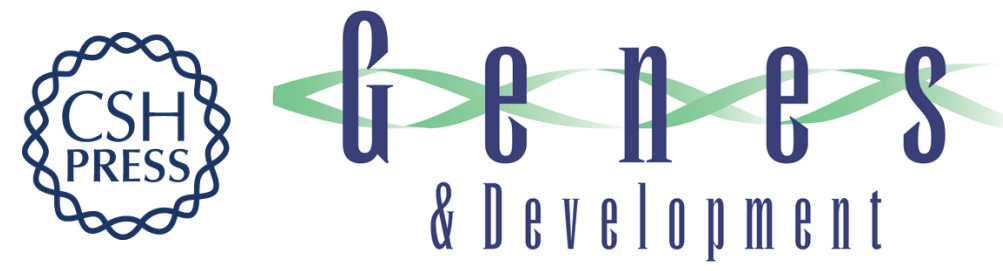

\section{A functional genomics strategy reveals clockwork orange as a transcriptional regulator in the Drosophila circadian clock}

Akira Matsumoto, Maki Ukai-Tadenuma, Rikuhiro G. Yamada, et al.

Genes Dev. 2007, 21: originally published online June 19, 2007

Access the most recent version at doi:10.1101/gad.1552207

\section{Supplemental http://genesdev.cshlp.org/content/suppl/2007/06/19/gad.1552207.DC1 Material}

References This article cites 50 articles, 20 of which can be accessed free at: http://genesdev.cshlp.org/content/21/13/1687.full.html\#ref-list-1

\section{License}

Email Alerting

Receive free email alerts when new articles cite this article - sign up in the box at the top Service

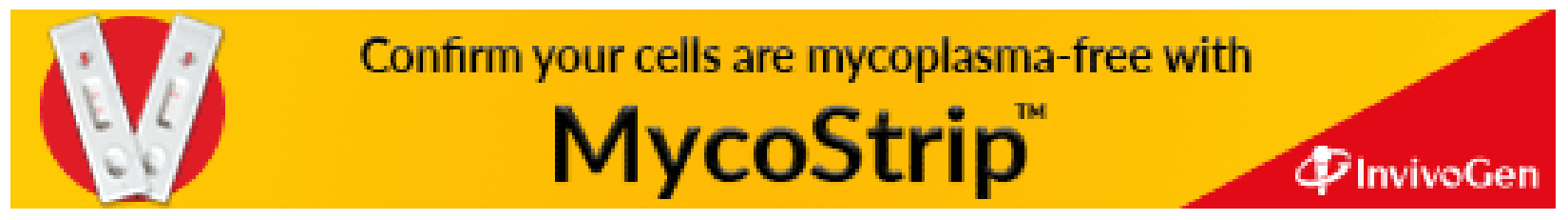

